\title{
Numerical modelling of \\ electromagnetic turbulent transport \\ of energetic ions in burning plasmas
}

\section{M.Albergante}

J. P. Graves, A. Fasoli, M. Jucker, X. Lapillonne and W. A. Cooper

Ecole Polytechnique Fédérale de Lausanne (EPFL)

Centre de Recherches en Physique des Plasmas (CRPP)

Association Euratom-Confédération Suisse

$\mathrm{CH}-\mathrm{IOI} 5$ Lausanne, Switzerland 


\section{Motivation}

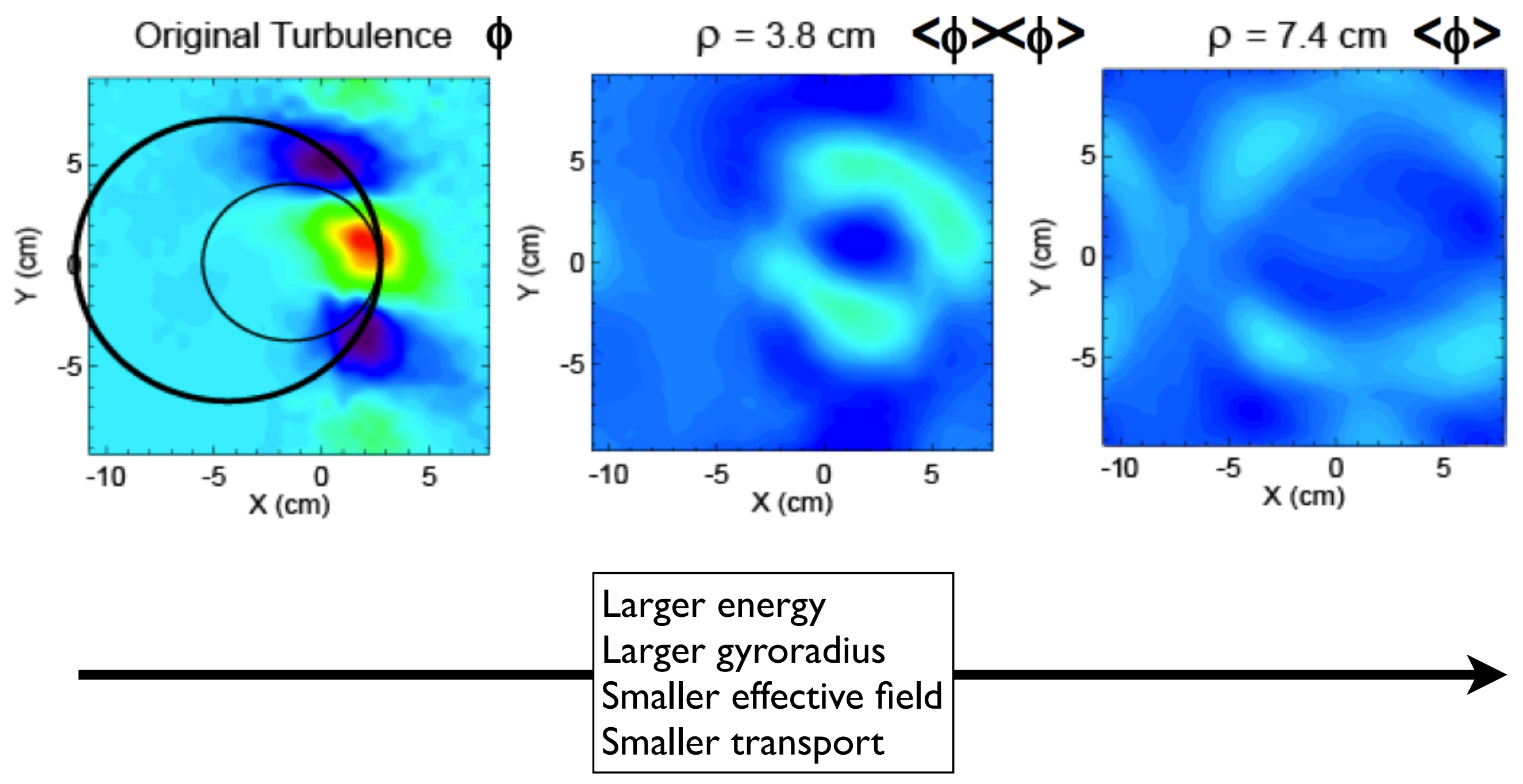

B. Heidbrink, GSEPWorkshop (20I0) 


\section{Motivation}
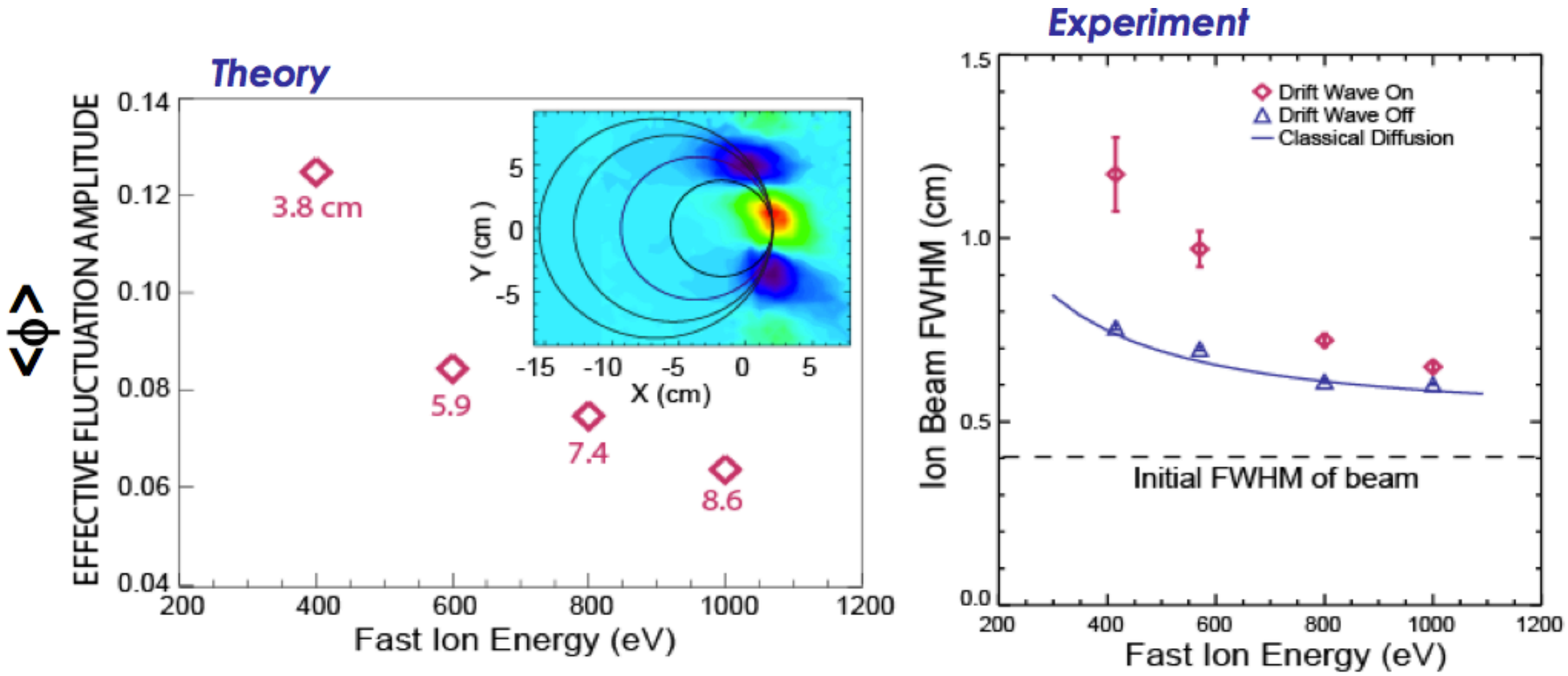

Larger energy

Larger gyroradius

Smaller effective field

Smaller transport

B. Heidbrink, GSEPWorkshop (20I0) 


\section{Motivation}

\section{ASDEX-U Experiments}

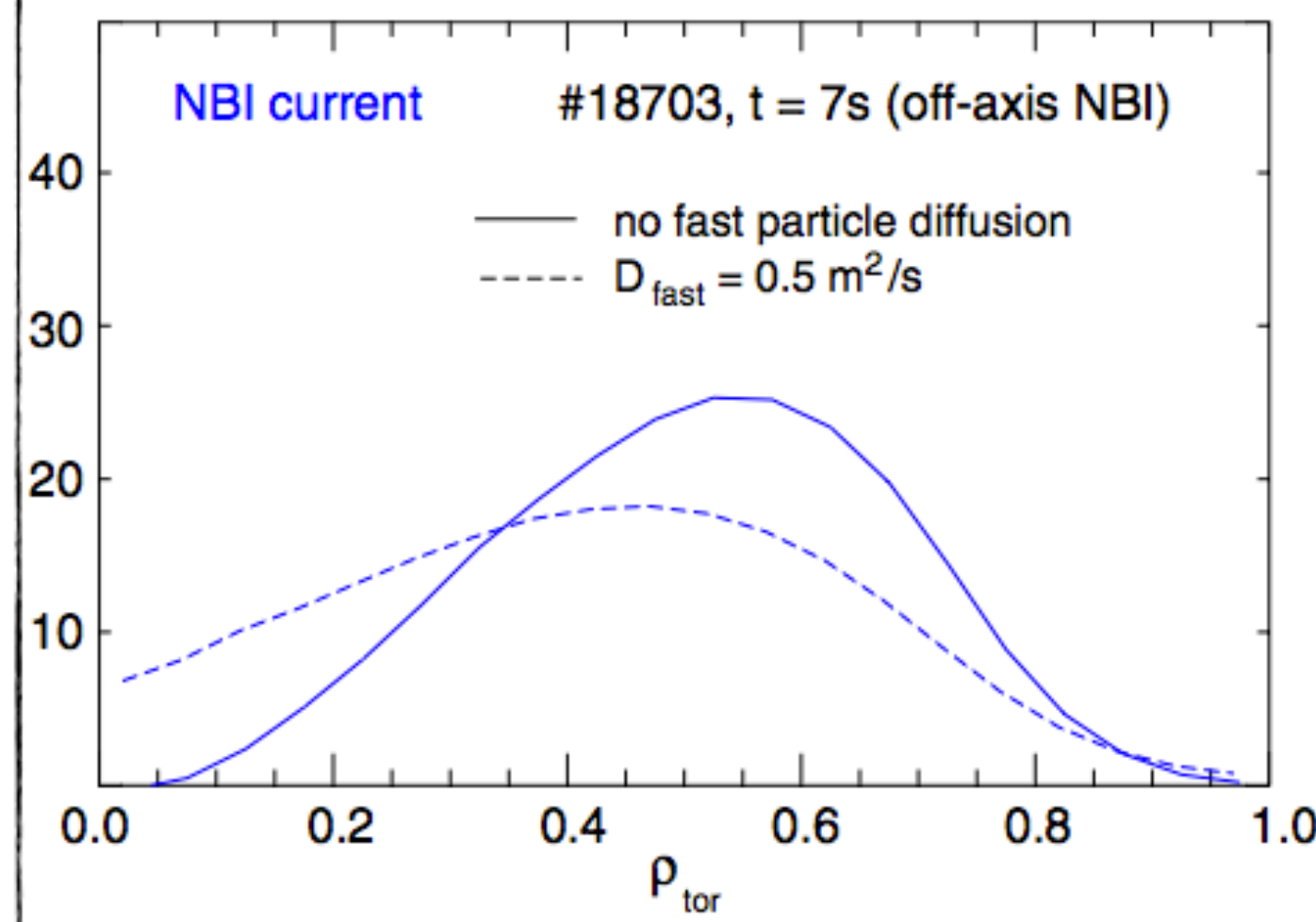

\section{DIII-D Experiments}

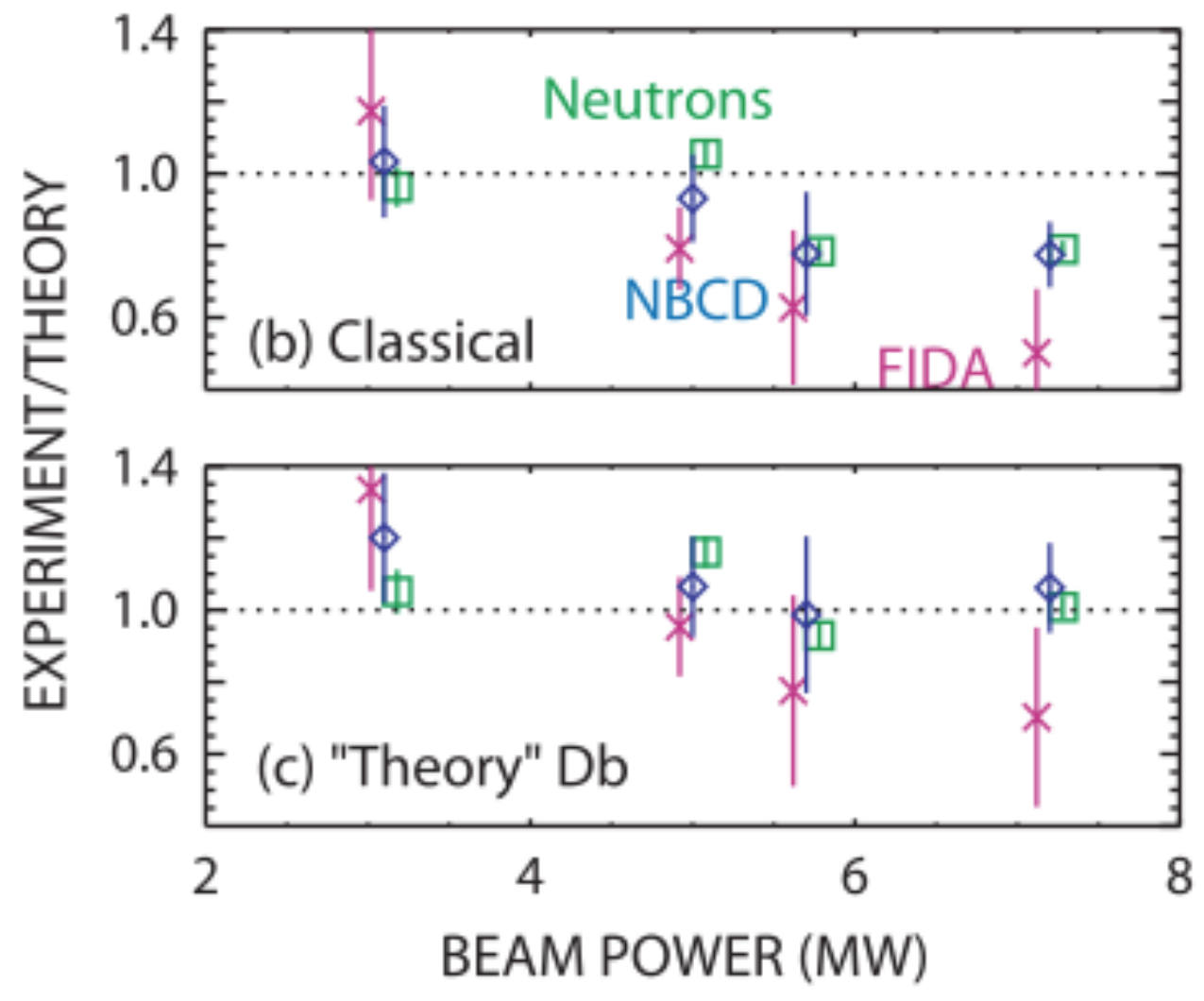

S. Günter, Nuclear Fusion (2007)

B. Heidbrink, Physical Review Letters (2010) 


\section{Motivation}

- Anomalies in NBI particles

- Smaller $E_{n b i} / T_{e}$ ratio

- larger transport

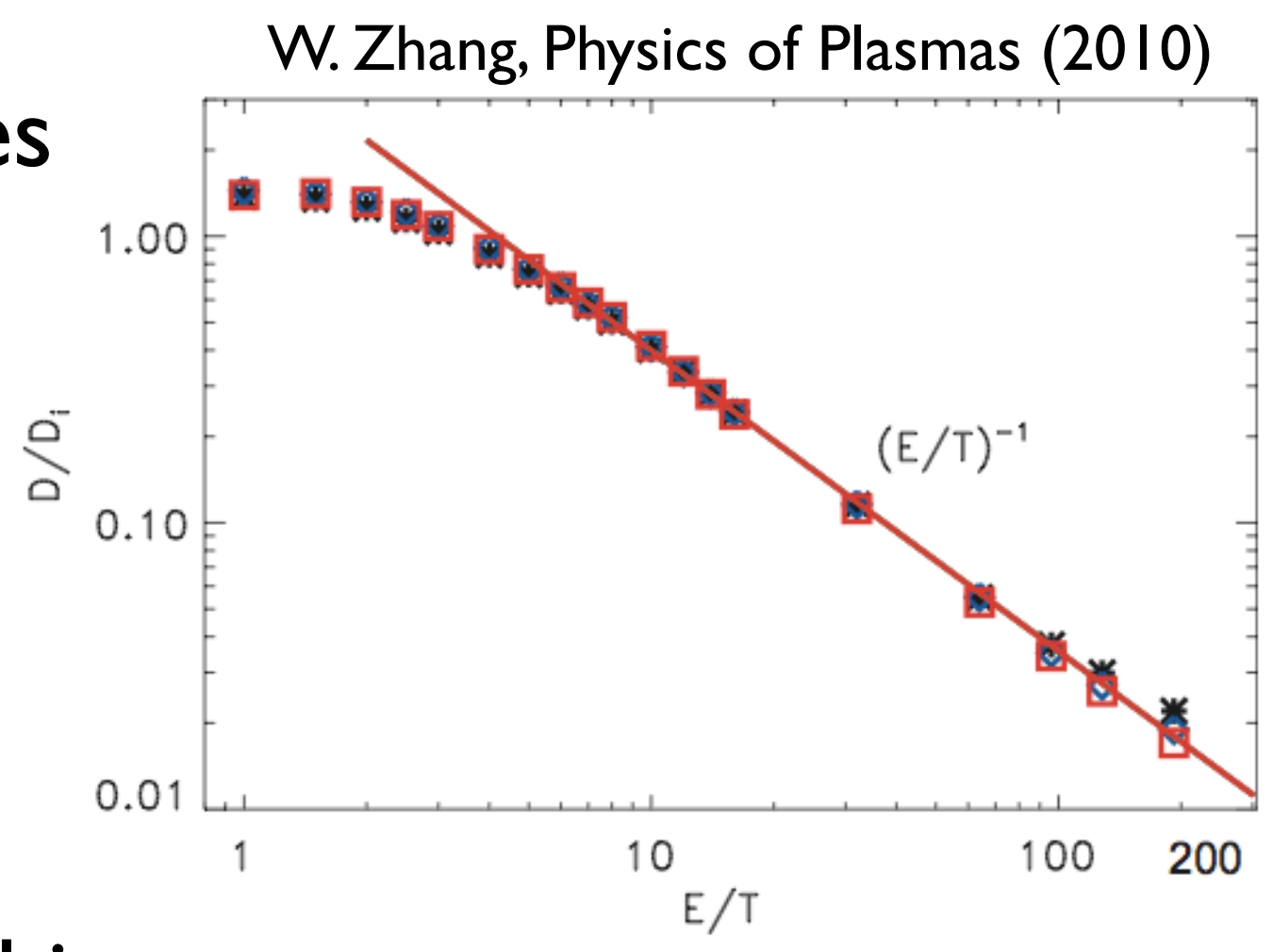

- Beam energies unchanged in past years

- Plasma temperatures larger in present day experiments 


\section{Motivation}

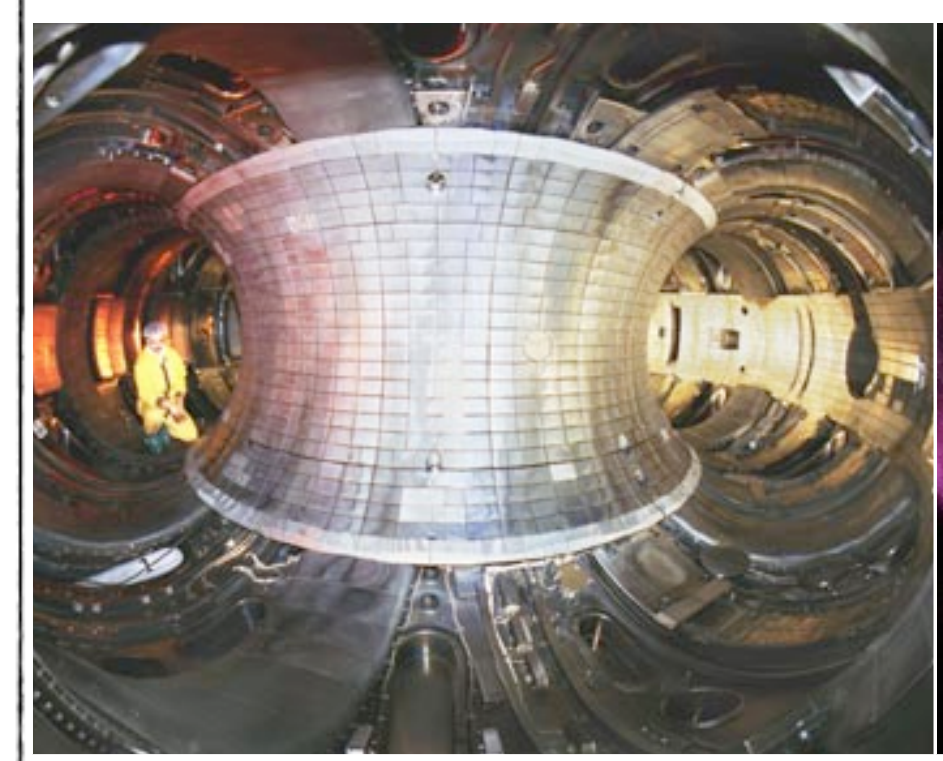

TFTR

Early Experiments

$E_{\alpha} / T_{e}=1000$

$E_{n b i} / T_{e}>30$

Neoclassical behaviour

C. Angioni, Nuclear Fusion

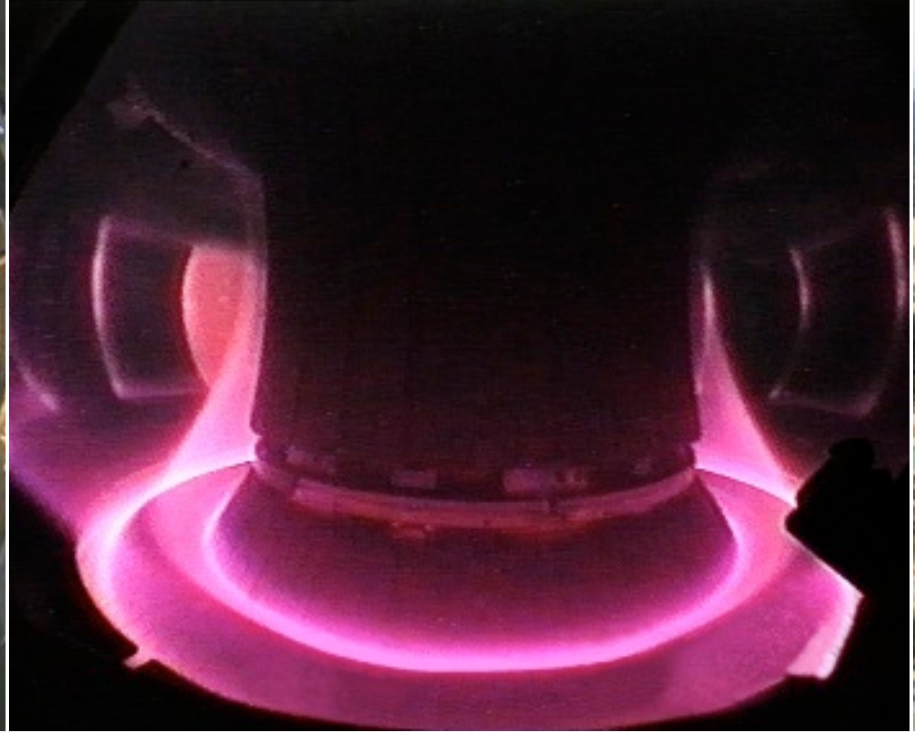

ASDEX Upgrade/DIII-D

Present day experiments Future Experiments

$E_{\alpha} / T_{e}>300$

$E_{n b_{i}} / T_{e} \leq 20$

Presence of anomalies
$E_{\alpha} / T_{e}>100^{*}$

$\mathrm{E}_{\mathrm{nbi}} / \mathrm{T}_{\mathrm{e}} \cong 30$

What effect on the NBI? 


\section{Outline}

Microturbulence

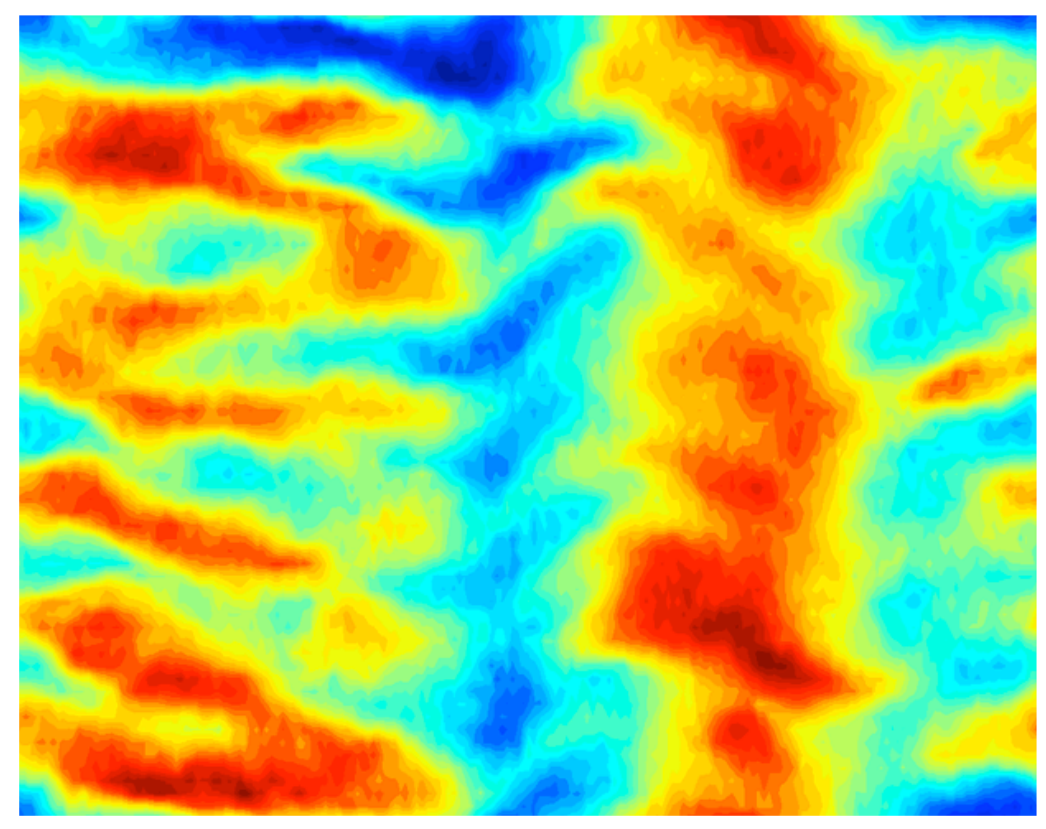

GENE code

ITER steady state

Energetic ion diffusivity
Neutral Beam Modelling

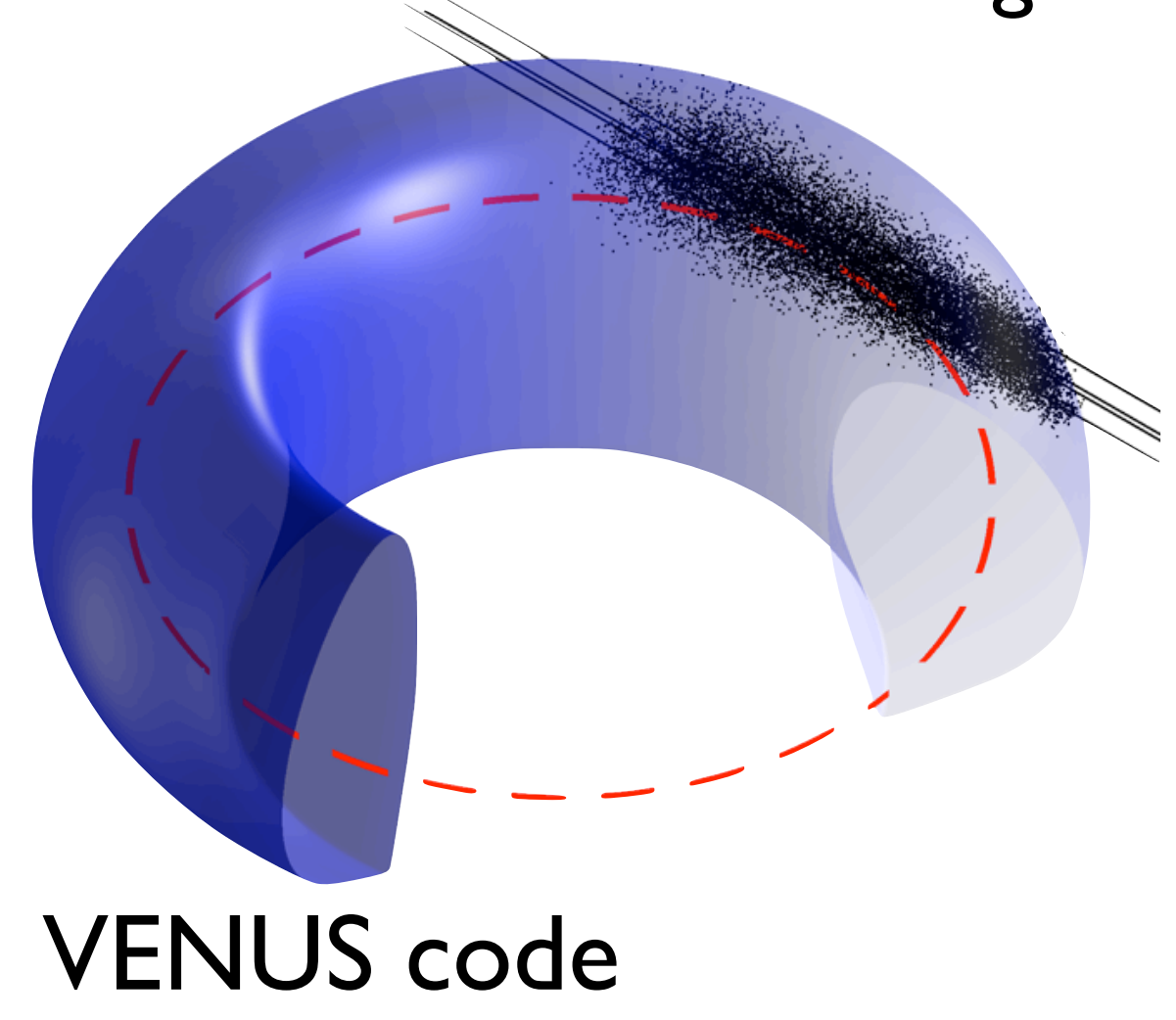

Collisional slowing down

Anomalous transport 


\section{Gyrokinetic simulations of turbulent transport}




\section{The numerical platform}

- GENE' code

- Linear and nonlinear flux tube simulations

- Electromagnetic perturbations

- Multi-species

- Interface with MHD equilibrium code $\mathrm{CHEASE}^{2}$

'F. Jenko, Physics of Plasmas (2000)

${ }^{2} \mathrm{H}$. Lütjens, CPC (1996) 


\section{Linear analysis}

- ITER steady state scenario (D+e $\left.e^{-}\right)$

- Simulations near marginal stability (at mid-radius)

- Temperature gradient

$\Omega_{T}=-\left(R_{0} / a\right) \mathrm{d} \ln T / \mathrm{d} \rho_{t}$

- Beta effects

$$
\beta_{e}=n_{e} T_{e} /\left(B_{0}^{2} / 2 \mu_{0}\right)
$$
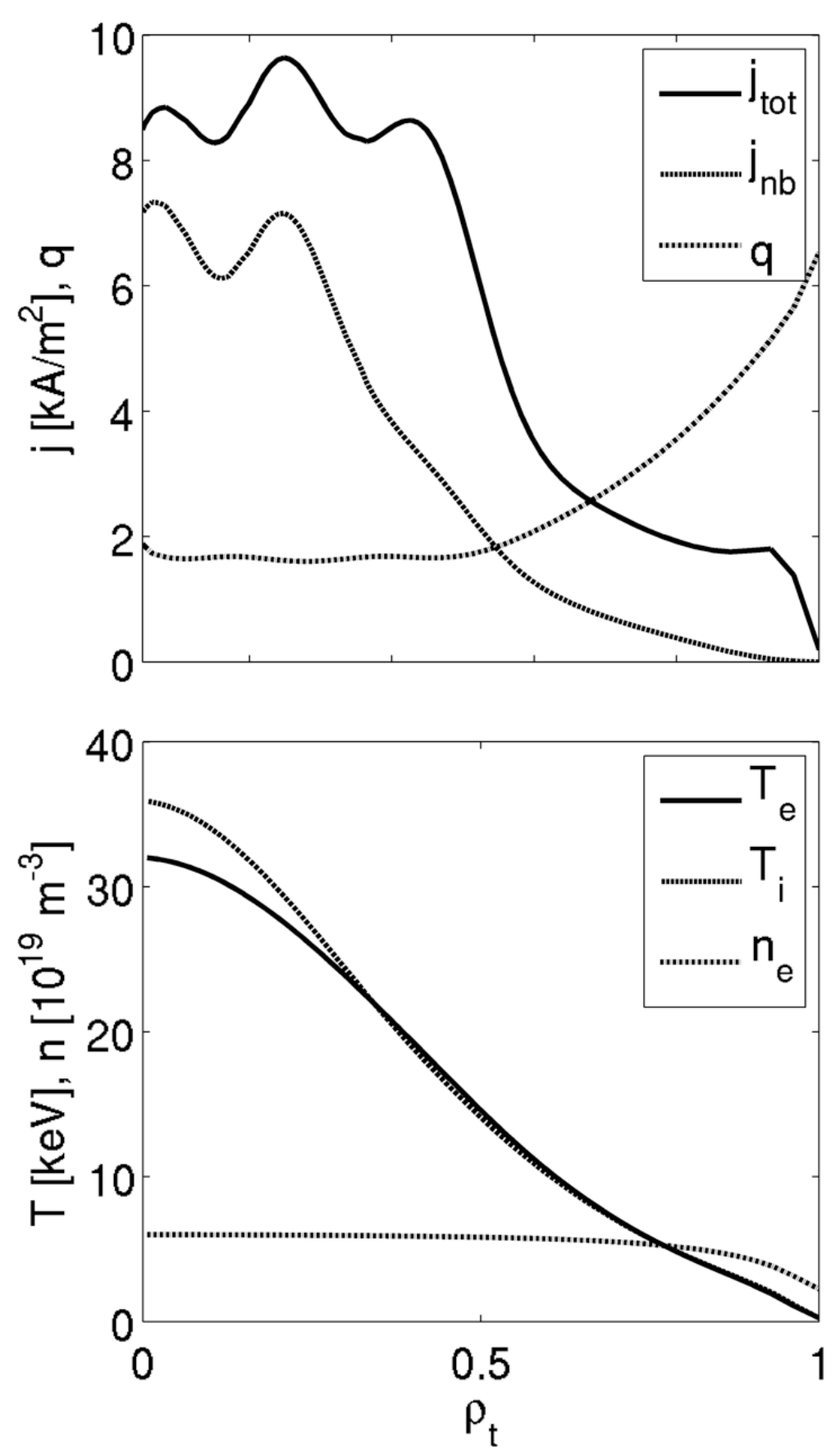


\section{Linear Analysis}

$$
\gamma\left[\mathrm{R}_{0} / \mathrm{c}_{\mathrm{si}}\right]
$$
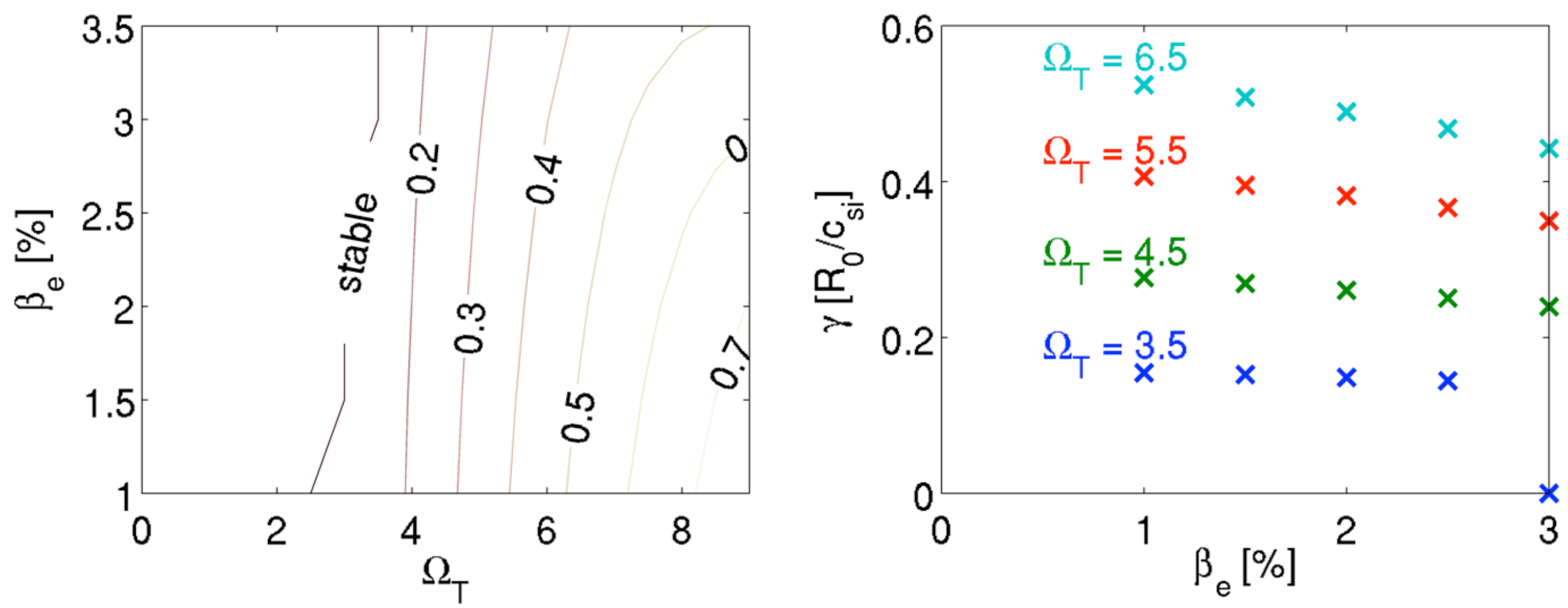

- ITG dominant instability

- Beta effects not exciting kinetic ballooning modes

- Subdominant modes are present

- Investigation for nonlinear simulation 


\section{Linear Analysis}

$$
\gamma\left[\mathrm{R}_{0} / \mathrm{c}_{\mathrm{si}}\right]
$$
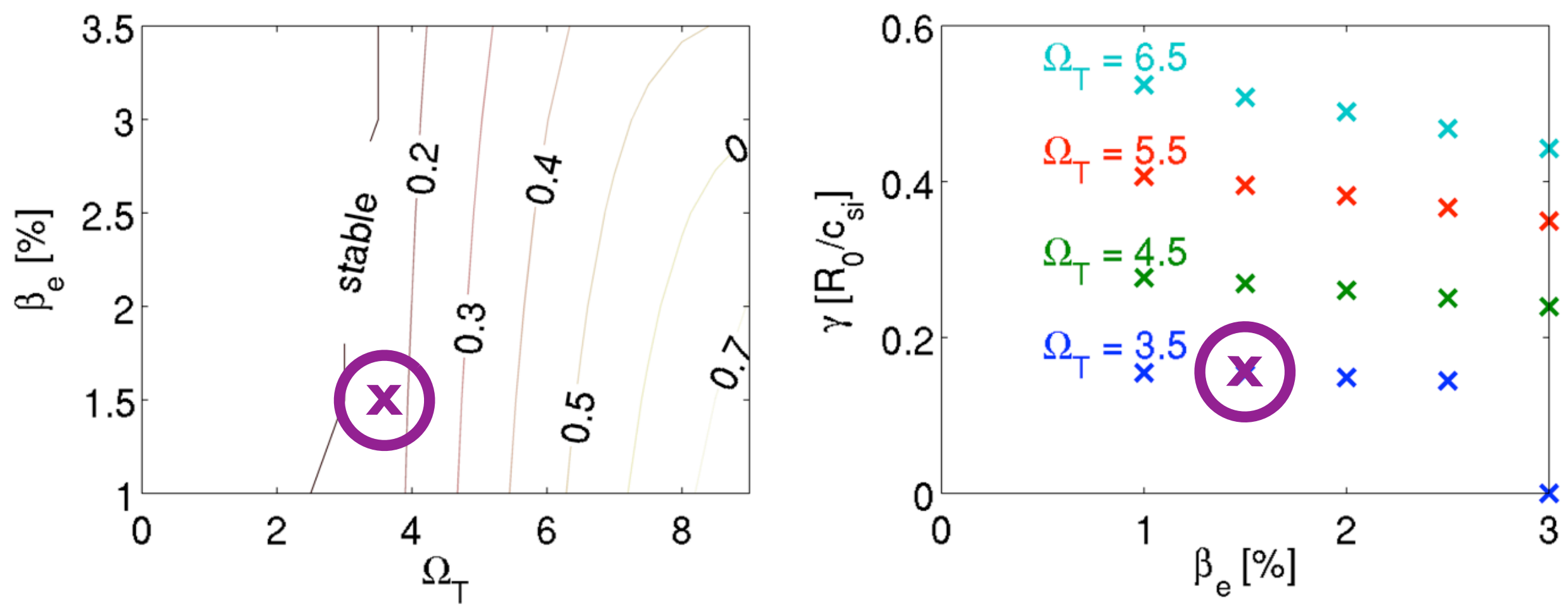

- ITG dominant instability

- Beta effects not exciting kinetic ballooning modes

- Subdominant modes are present

- Investigation for nonlinear simulation 


\section{Linear Analysis}

- Turbulence

- Dominant ITGs

- Subdominant TEMs

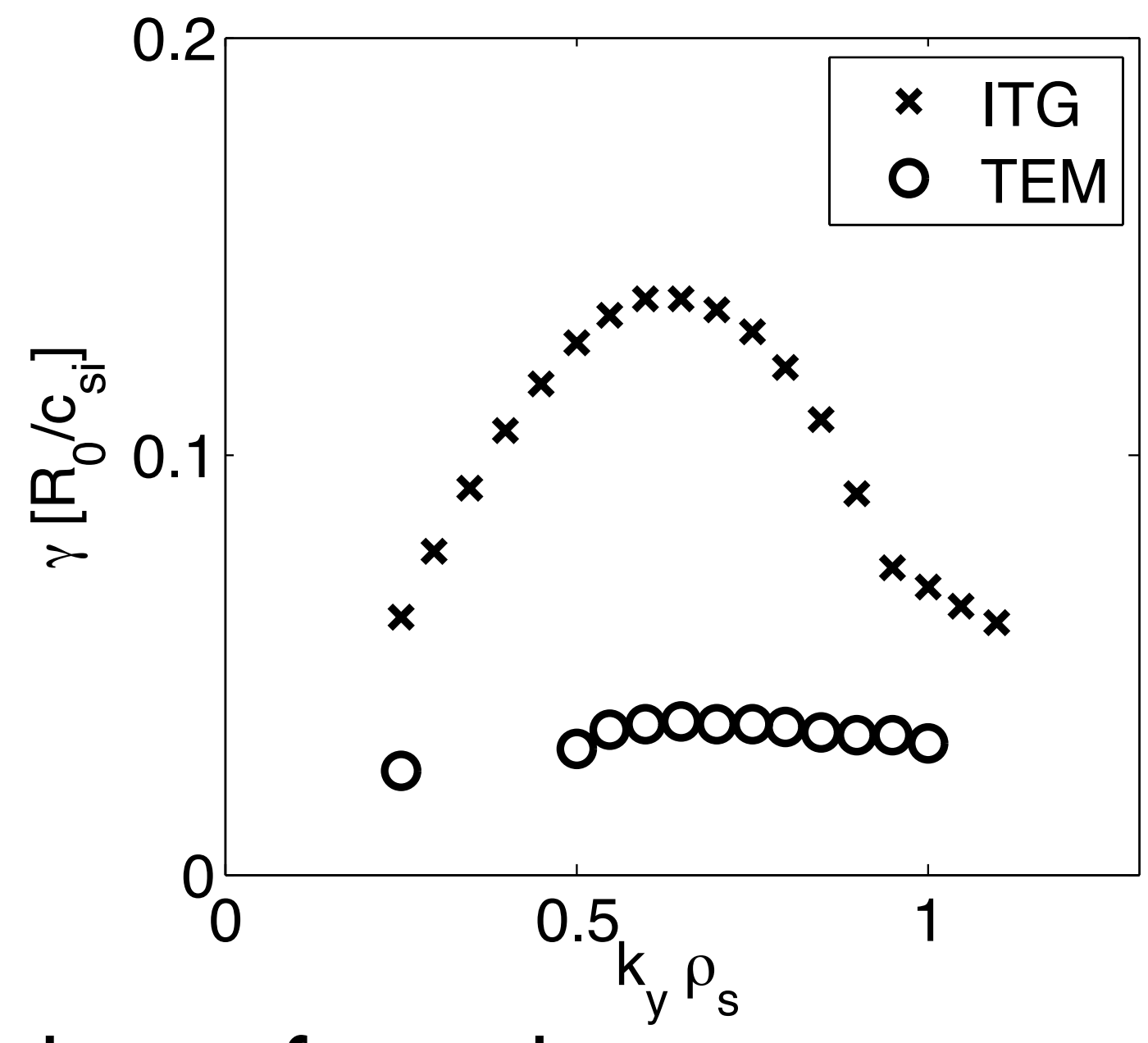

- Nonlinear simulations can be performed 


\section{Nonlinear Analysis}

- Mixture of ITG and TEM

- Magnetic perturbations

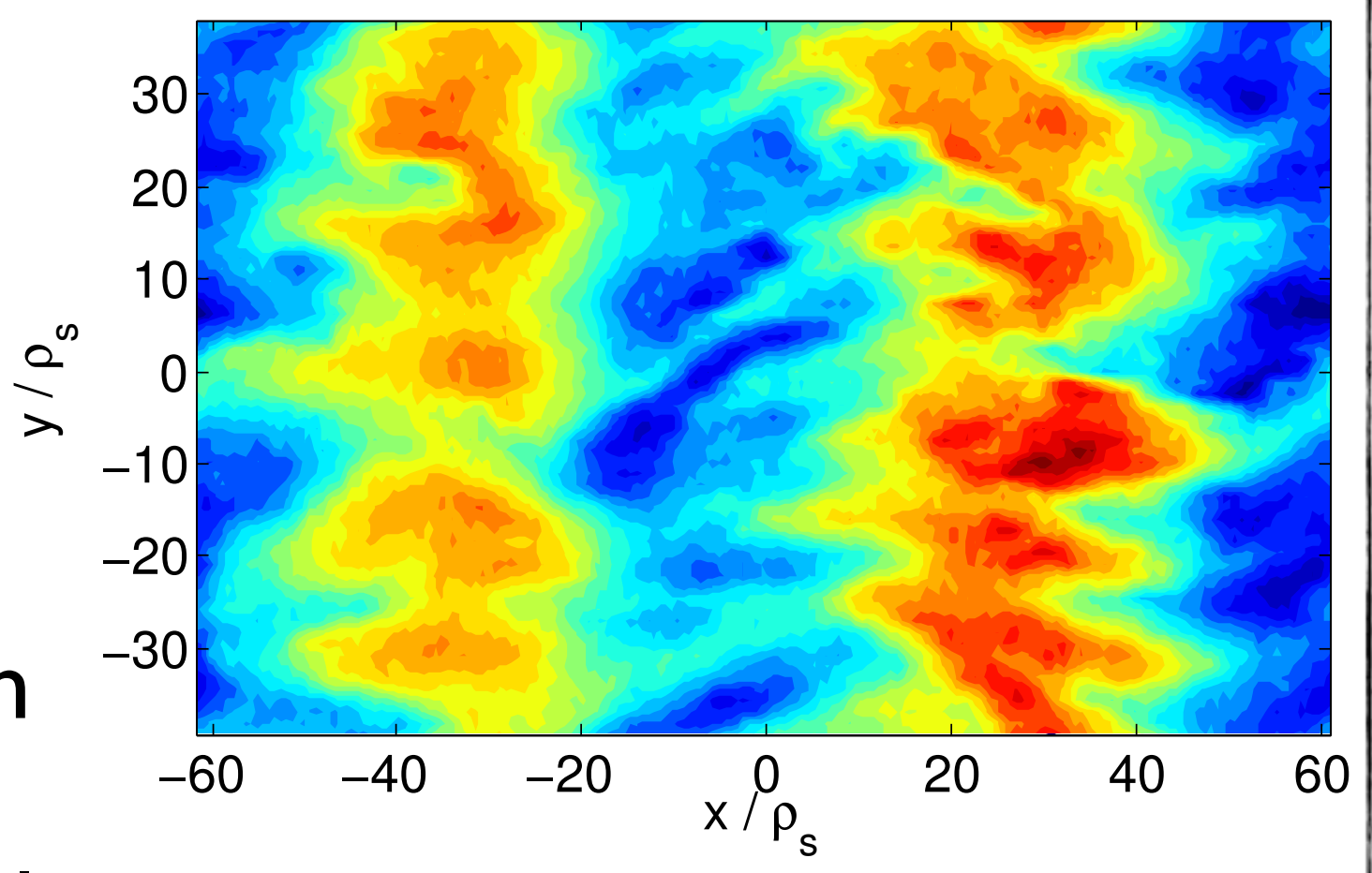

- Beam ions non-thermal

- Passive deuterium

- Maxwellian distribution

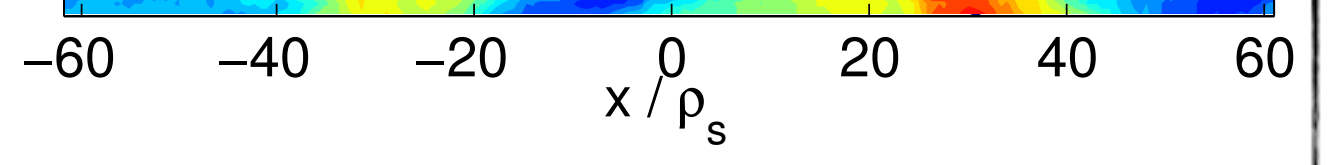

- What variables can describe the particle diffusivity? 


\section{The variables studied}

- Energetic particle transport is a diffusive process*

- It must be consistent with Fick's law

$$
D(\mathbf{x})=-\frac{\Gamma(\mathbf{x})}{\nabla n(\mathbf{x})}=-\frac{1}{\nabla n(\mathbf{x})} \int \delta f(\mathbf{x}, \mathbf{v}) \delta u(\mathbf{x}, \mathbf{v}) d \mathbf{v}
$$

- Allows for 'electrostatic' and 'magnetic' transport separation

$$
\delta \mathbf{u}^{e s}=-\frac{\nabla \delta \bar{\Phi} \times \mathbf{B}}{B^{2}} \quad \delta \mathbf{u}^{e m}=v_{\|} \frac{\nabla \delta \bar{A}_{\|} \times \mathbf{B}}{B^{2}}
$$




\section{The variables studied}

- Velocity space resolved diffusivity (gyroaveraged)

$$
D_{v}(\mathbf{x}, \mathbf{v})=-\frac{1}{\nabla \ln n(\mathbf{x})} \frac{\delta f(\mathbf{x}, \mathbf{v})}{f_{0}(\mathbf{x}, \mathbf{v})} \delta \mathbf{u}(\mathbf{x}, \mathbf{v}) \cdot \hat{\mathbf{e}}_{r}
$$

- Consistent with Fick's law

$$
\left\langle D_{v}\right\rangle_{f_{0}}=\frac{\int d \mathbf{v} D_{v}(\mathbf{x}, \mathbf{v}) f_{0}}{\int d \mathbf{v} f_{0}}=-\frac{\Gamma(\mathbf{x})}{\nabla n(\mathbf{x})}=D^{\mathrm{eff}}(\mathbf{x})
$$




\section{Nonlinear results}

$\mathrm{D}_{\text {es }}\left[\mathrm{m}^{2} / \mathrm{s}\right]$ at $\theta=0$

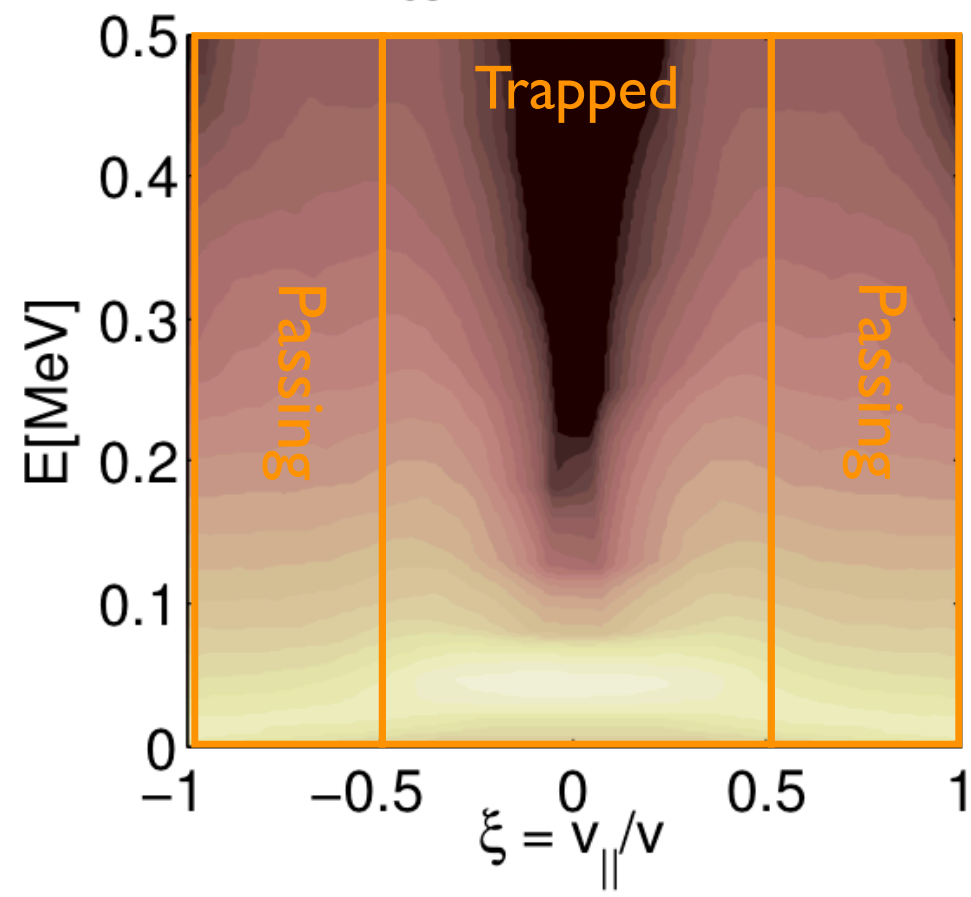

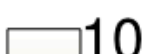

$10^{-1}$

$10^{-2}$

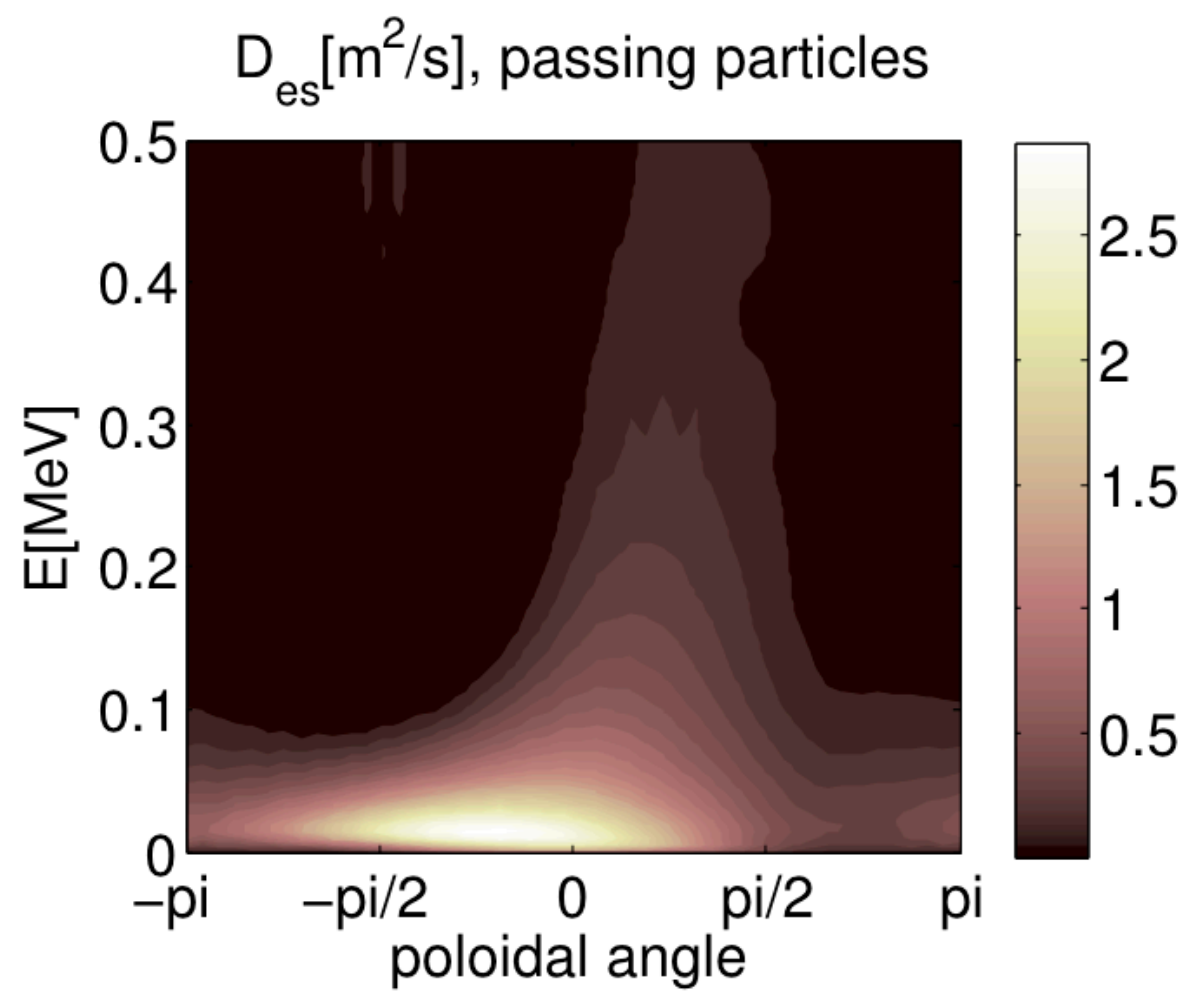

- Trapped ions: orbit- and gyro-averaging

- Passing ions: no gyro-averaging, orbit-averaging?

- Above collisional estimates ( $(-)$ 


\section{Transport Summary}

- Potentially large electrostatic transport for beam ions

- Magnetic transport negligible

- What impact on the beam driven current?

- Can poloidal effects/collisions play a role? 
Simulation of the neutral beam injection and slowing down 


\section{NBI Modelling}

(I) 4 Injectors

(2) Tangential

(3) Uniform number of particles

(4) Beam collimation reproduced

(5) Parallel velocity from CHEASE

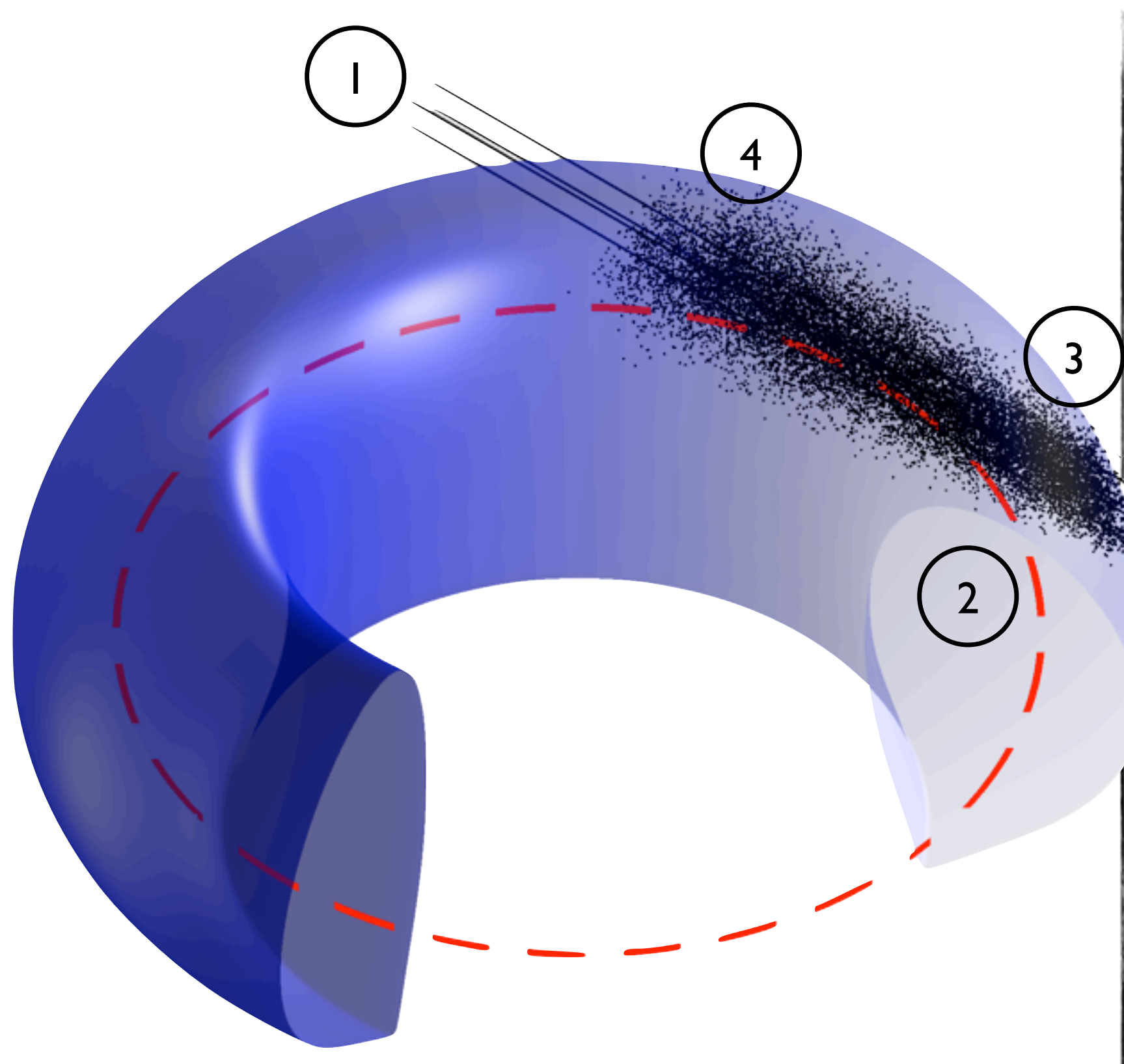

(6) What weight? 


\section{NBI Geometry}

- Beam intensity

$$
I(\ell)=I_{0} e^{-\int_{0}^{\ell} n_{e}\left(\ell^{\prime}\right) \sigma_{\text {eff }}\left(\ell^{\prime}\right) d \ell^{\prime}}
$$
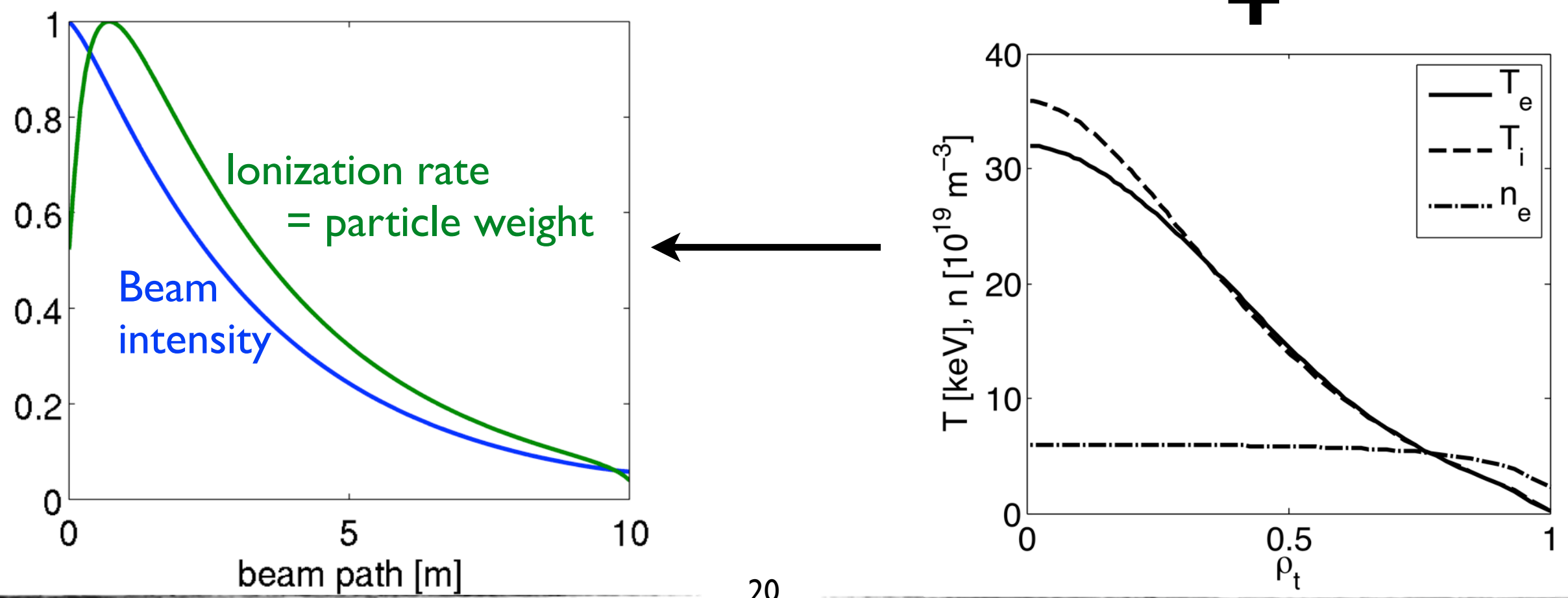


\section{NBI Geometry}

- Beam intensity

$$
I(\ell)=I_{0} e^{-\int_{0}^{\ell} n_{e}\left(\ell^{\prime}\right) \sigma_{\text {eff }}\left(\ell^{\prime}\right) d \ell^{\prime}}
$$
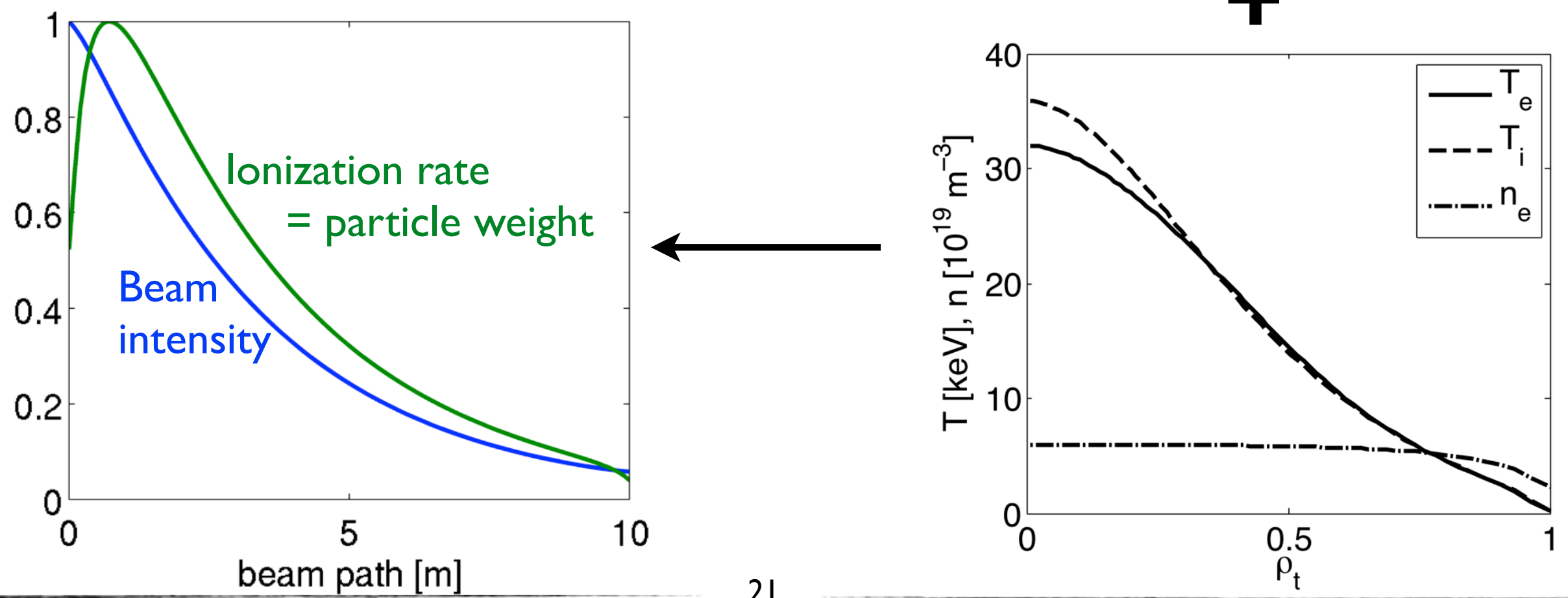


\section{NBI Geometry}

- Beam intensity

$$
I(\ell)=I_{0} e^{-\int_{0}^{\ell} n_{e}\left(\ell^{\prime}\right) \sigma_{\text {eff }}\left(\ell^{\prime}\right) d \ell^{\prime}}
$$
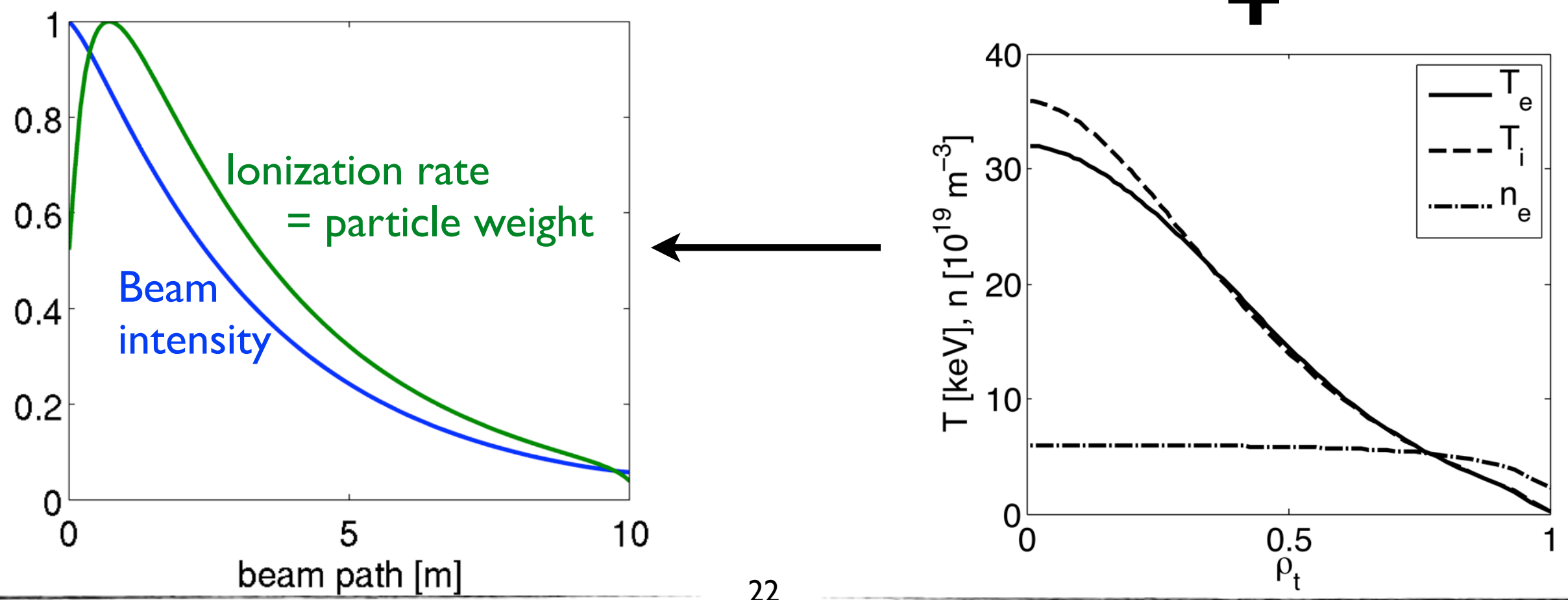


\section{$\mathrm{NBI}$ Geometry}

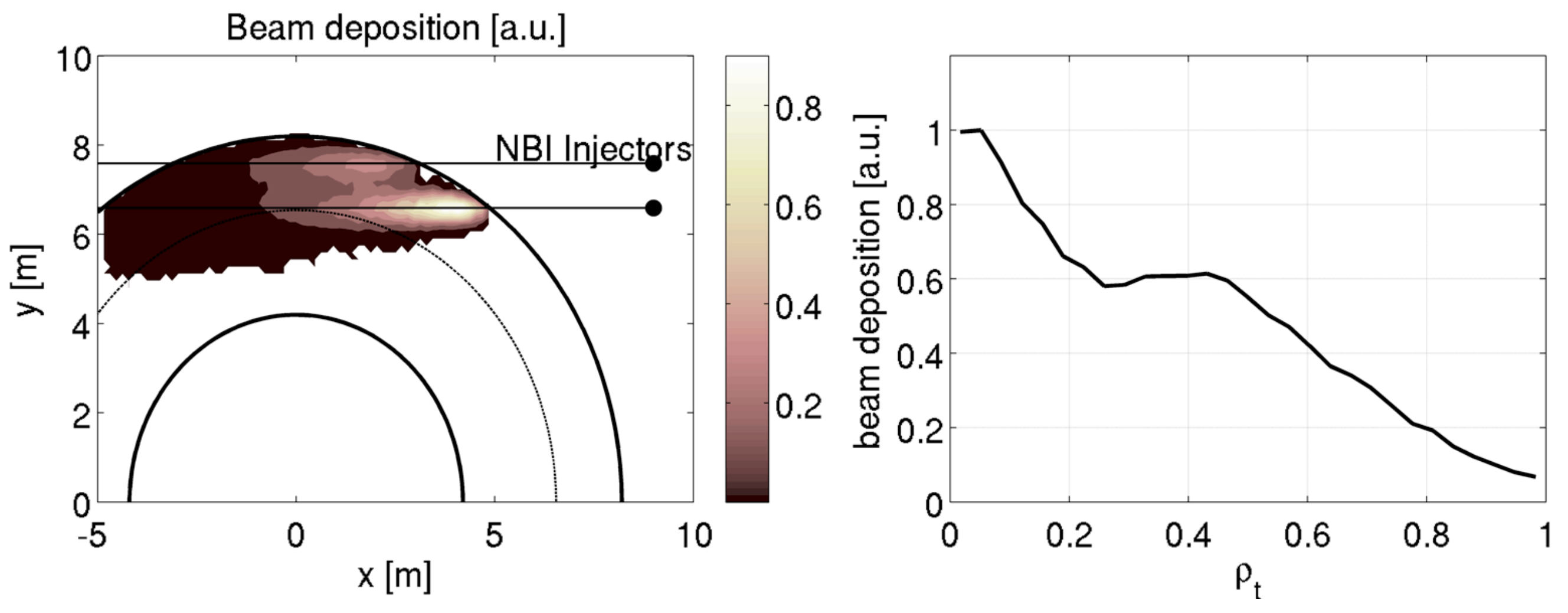

- Broad deposition, peaked profile

- Edge deposition: need for high energy NBI

- What time evolution? 


\section{The VENUS Code*}

- Drift-kinetic particle pushing code

- Velocity space kicks for Coulomb collisions

- Inclusion of electron drag

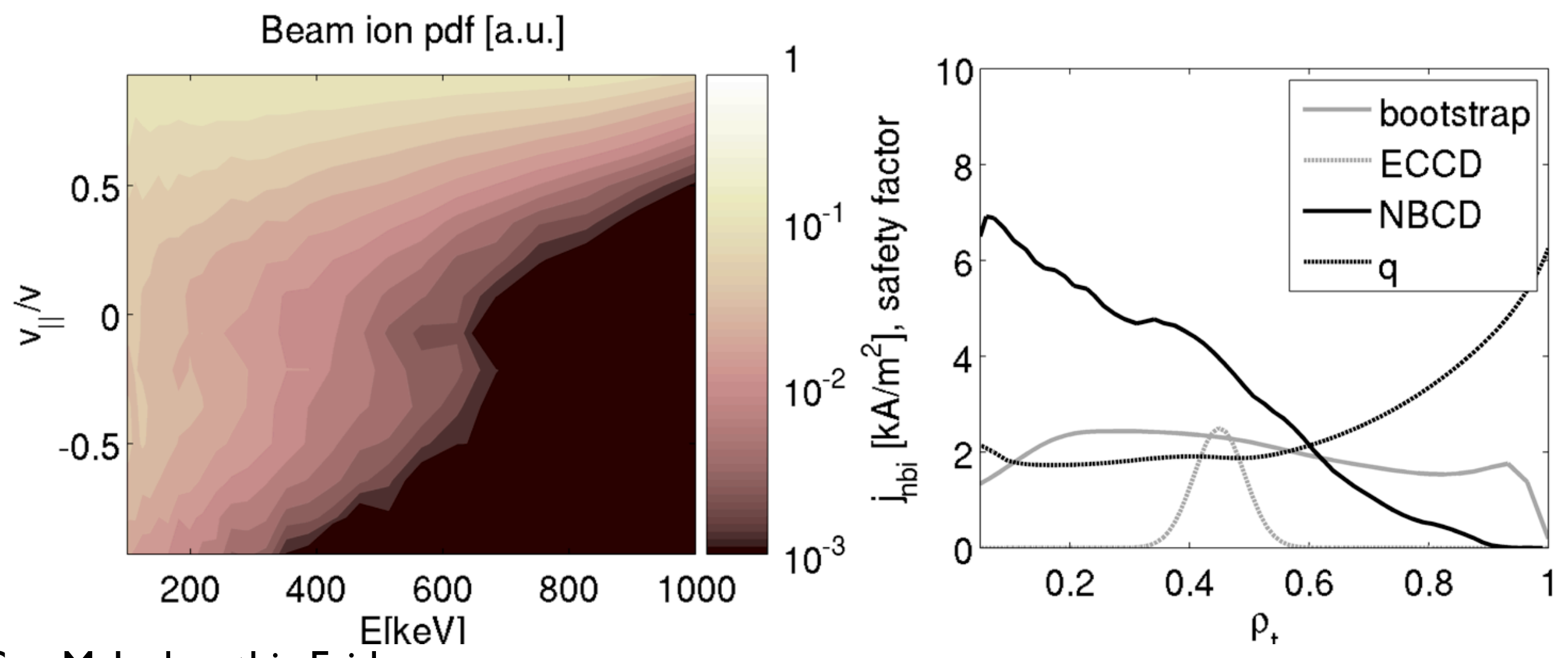




\section{$\mathrm{NBI}$ summary}

- Neutral beam model ready

- Collisional slowing down of NBI particles

- Anomalous transport must be implemented 


\section{Anomalous diffusivity module}

- Monte-Carlo diffusion

- Effective gyroaveraged diffusivity from GENE simulations

- Interpolate at particle position

- Radial envelope
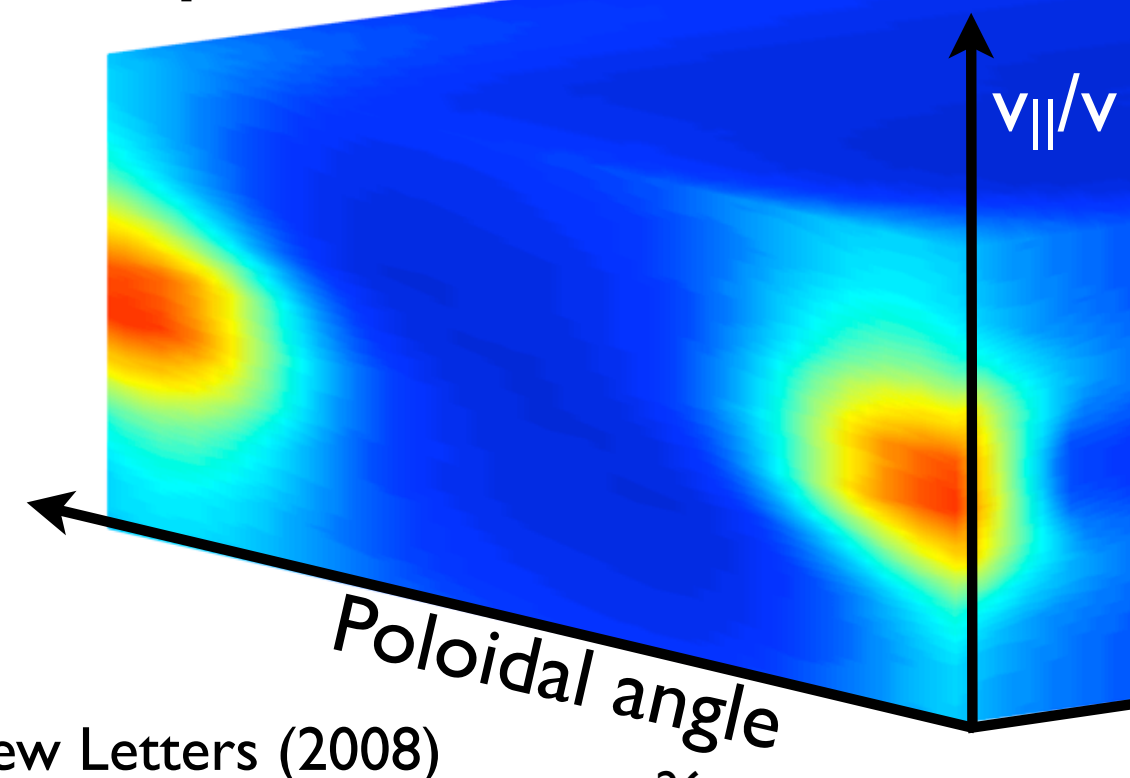


\section{Results}

- NBCD profile redistributed

- Small changes in the safety factor

- Moderate shear reversal

- 'Averaged' model is a good approximation

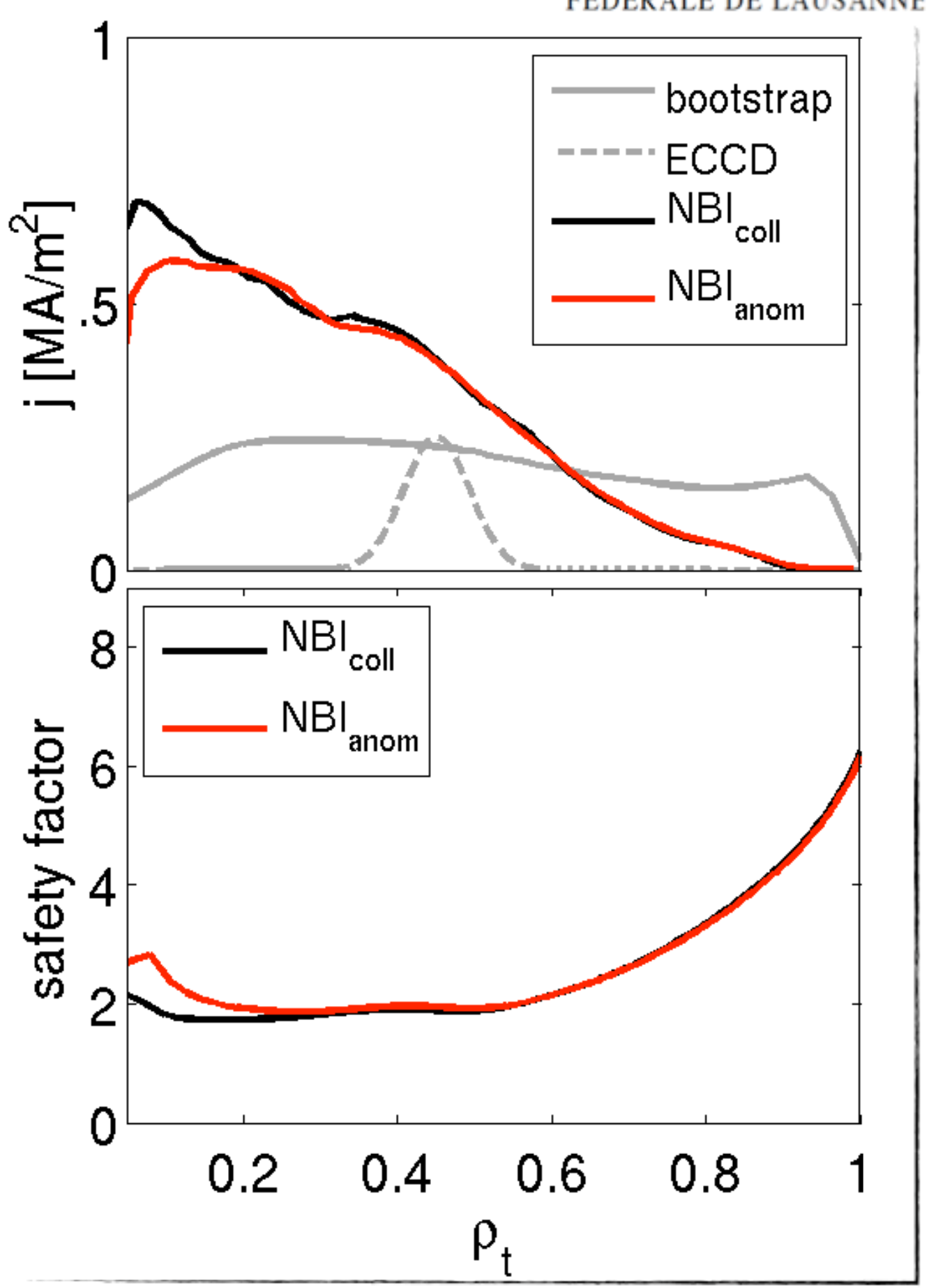




\section{Results}

- Main disadvantage

- Local approximation

- Solutions

- Global version of GENE*

- Multiple flux tube simulations ${ }^{+}$

- Low microturbulent impact. Why?

*T. Görler, tomorrow +M. Barnes, Thursday

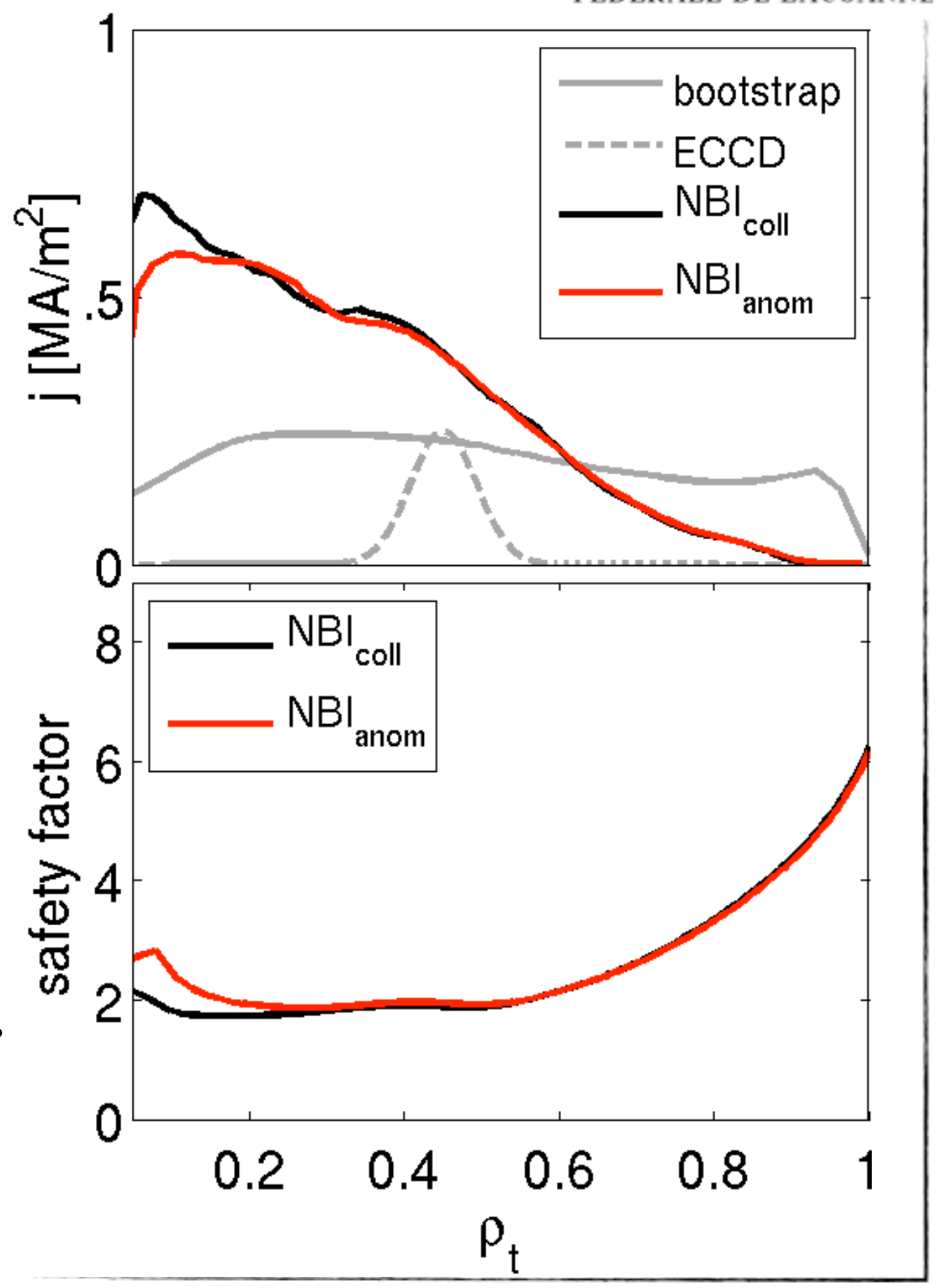




\section{Results}

- Low diffusivity for particles contributing to the current

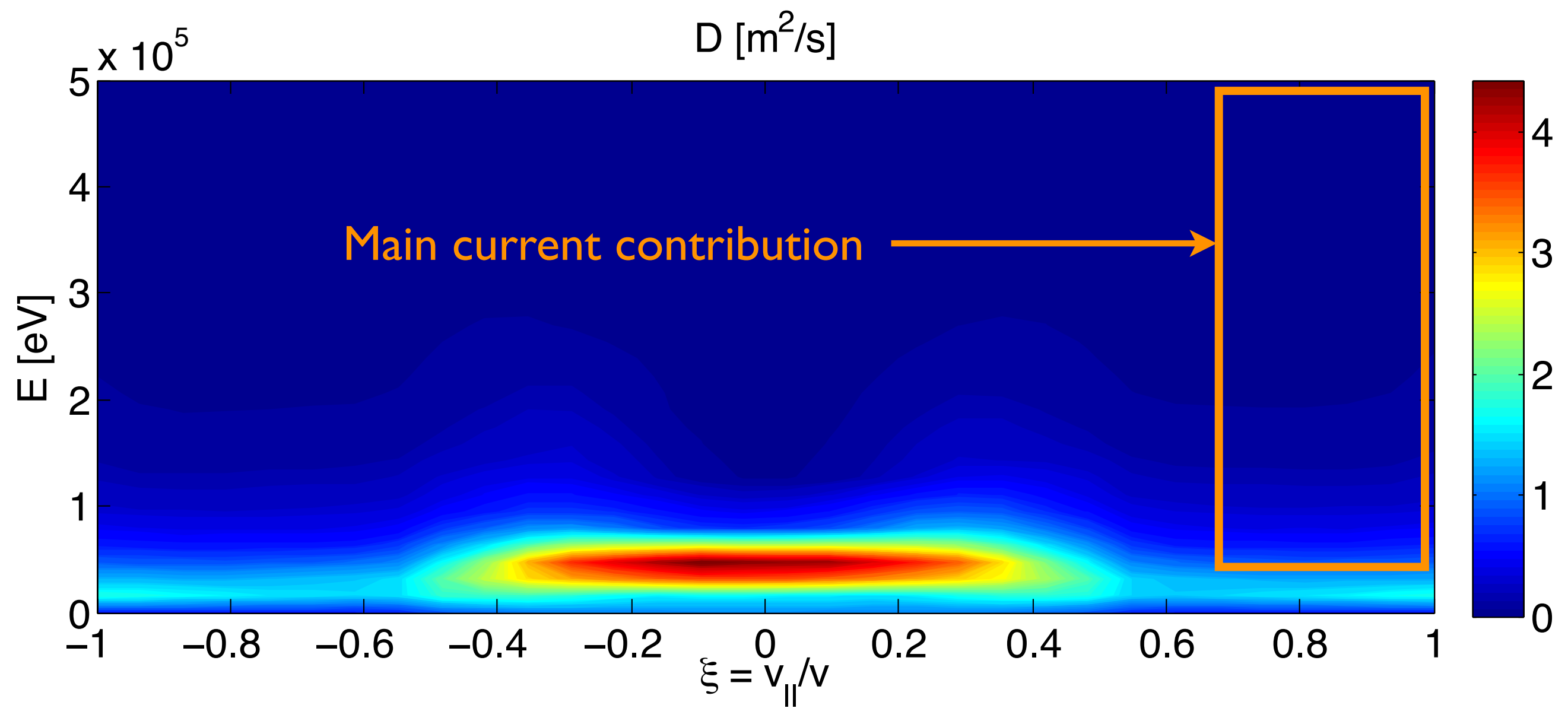

- High energy $\mathrm{NBI}$ at I MeV good choice 


\section{Lower energy NBI}

- $\mathrm{E}_{\mathrm{nbi}}=300 \mathrm{keV}$ at mid-radius

- $E_{n b i} / T_{e}=20$ (similar to ASDEX)

- Previous scenario $E_{n b i} / T_{e}>50$ at mid-radius

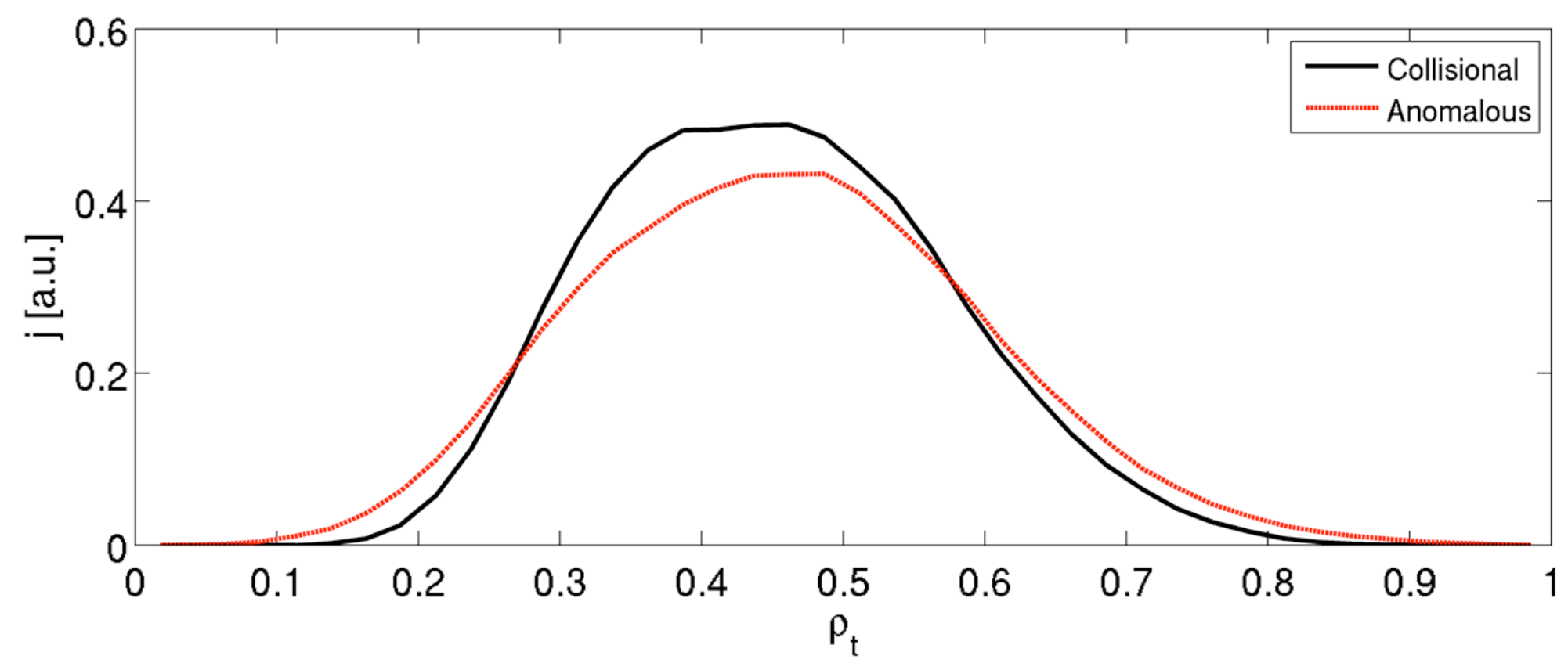

- Beam redistribution more important 


\section{Conclusions}

Microturbulence

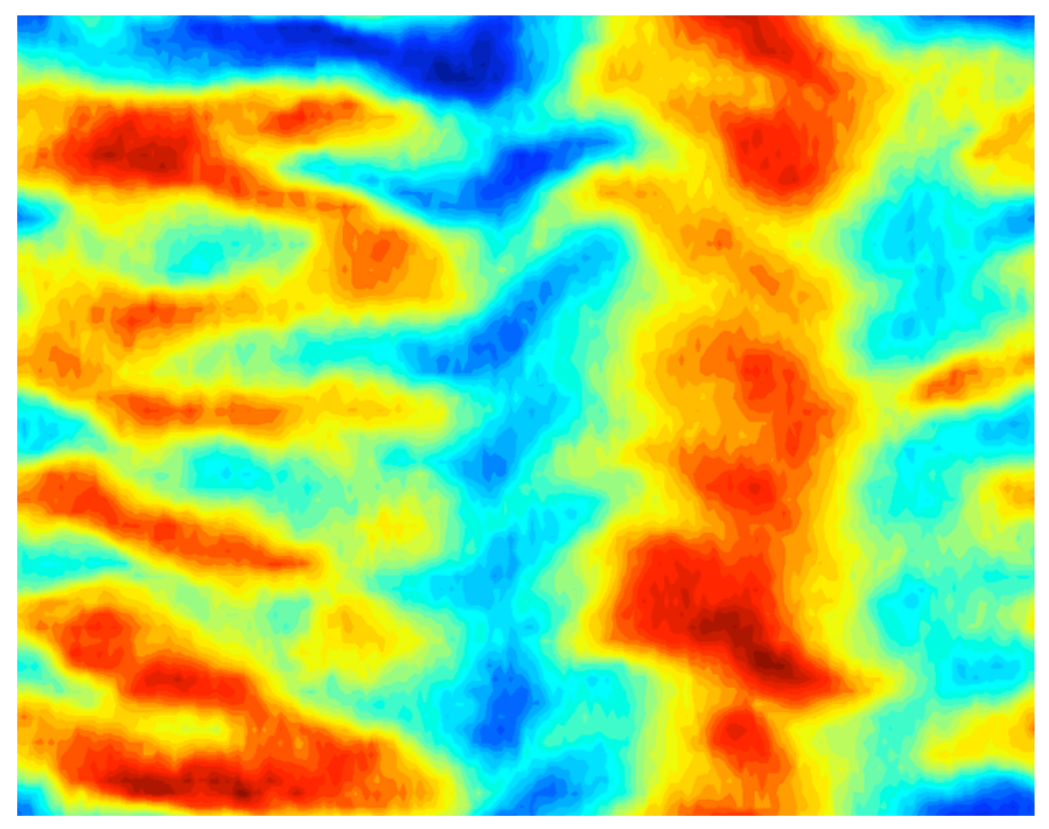

GENE code

Energetic ion diffusivity

Velocity space analysis
Neutral Beam Modelling

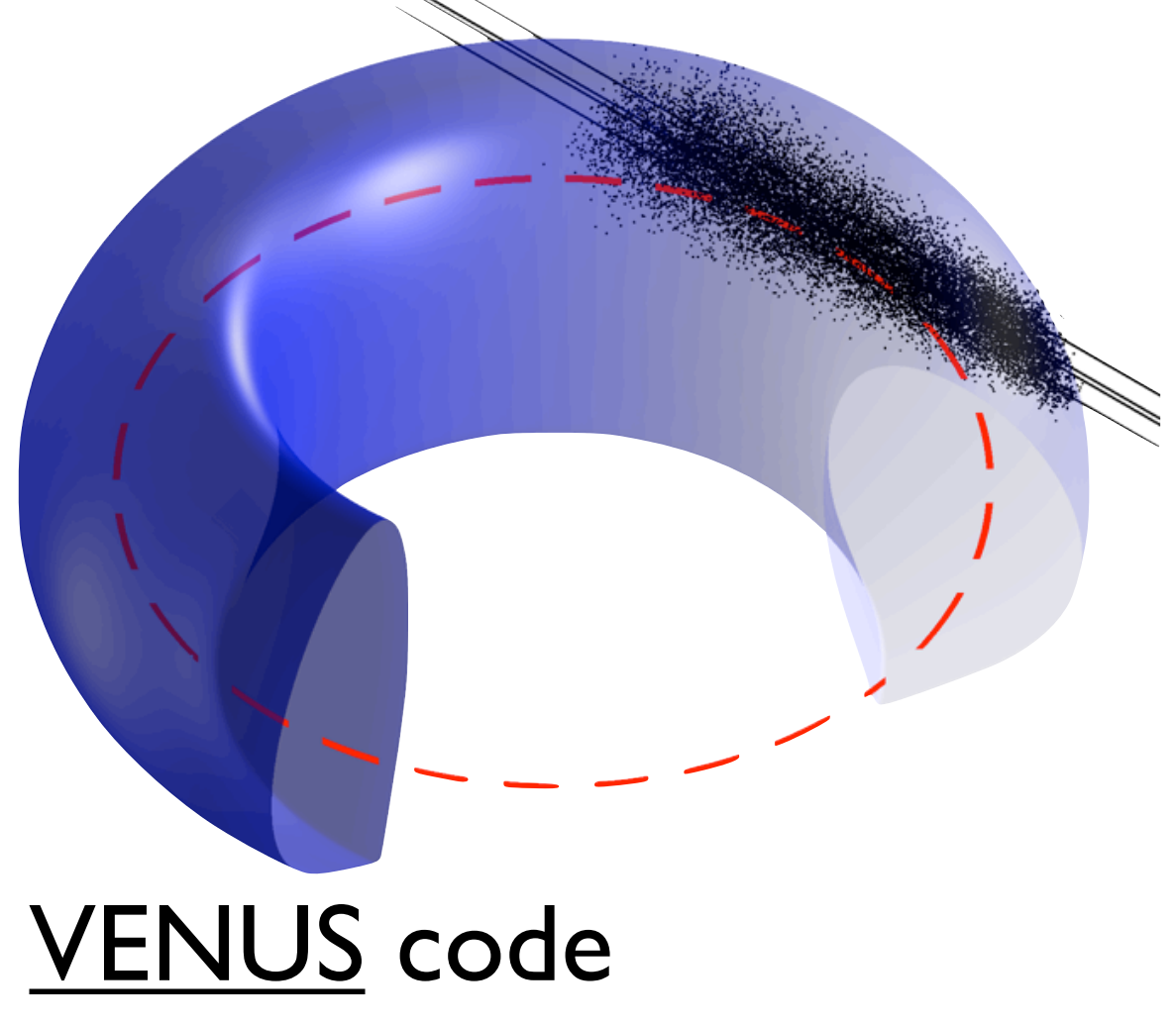

Collisional slowing down

Anomalous transport 


\section{Conclusions}

- Modelling of the ITER steady state scenario

- Consequences for I MeV NBI

- Small but potentially important NBCD redistribution

- Transport and stability would change

- Consequences for low energy NBI

- Larger transport, more redistribution

- Our model underestimate?

- NBI model improvements ( $E_{n b i} / 2$ and $E_{n b i} / 3$ fractions)

- Background turbulence potentially stronger 


\section{Outlook}

- DEMO reference scenarios more affected

- Large beam current (I MeV)

- Plasma temperature two times ITER's goal

- More detailed and self consistent turbulence

- very challenging

- Comparison with experimental data

- even more challenging 


\section{Linear Analysis}

- Subdominant TEMs

- Also investigated ETG

$$
\frac{\gamma^{E T G}}{\gamma^{I T G}} \ll \sqrt{\frac{m_{e}}{m_{i}}}
$$

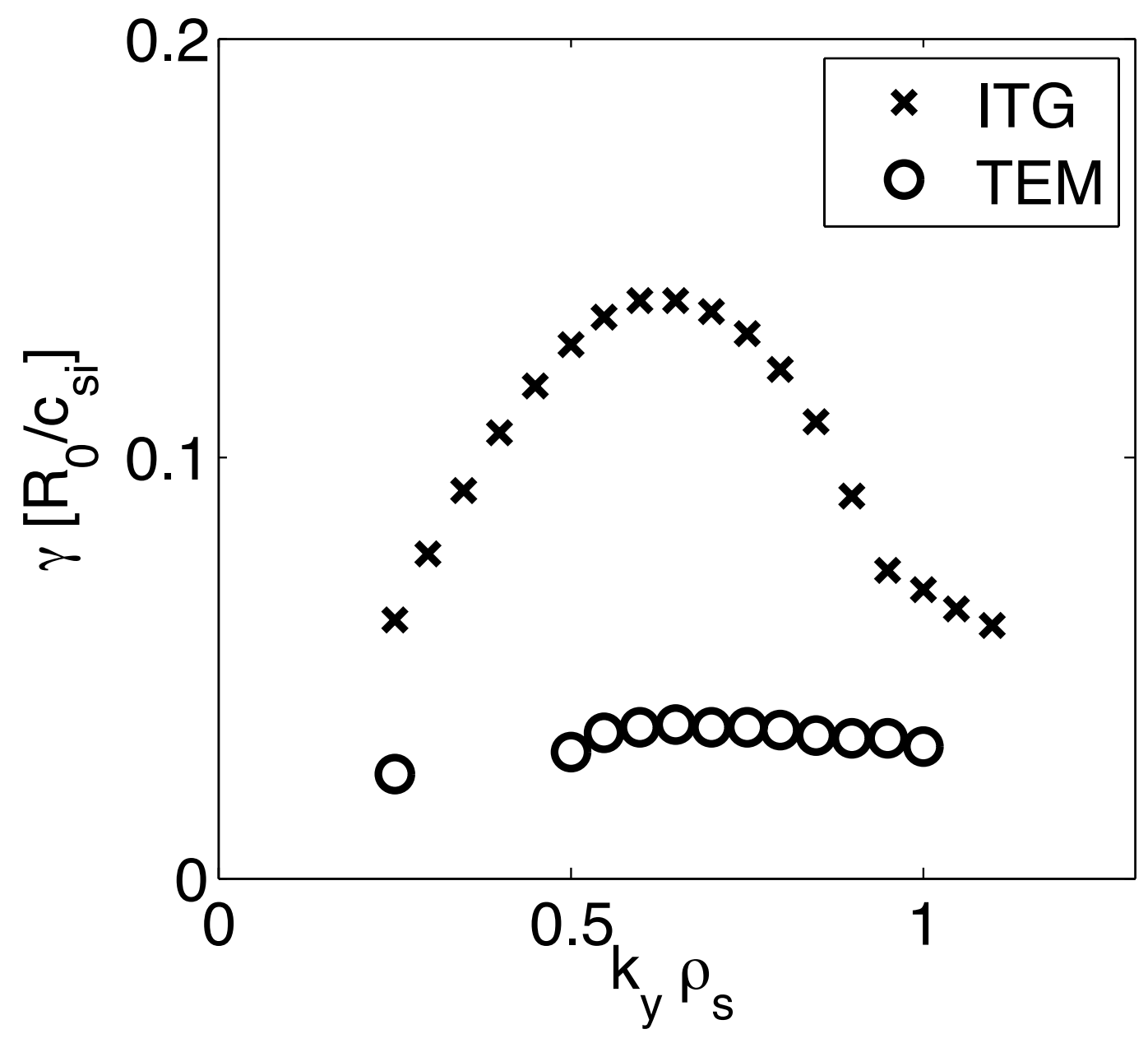

- Hyperfine scale neglected 


\section{Nonlinear analysis}

Parameters

[nx nky nz]

[ 1923248$]$

[nvpar nmu]

[64 32]

$\mathrm{ky}_{\mathrm{y} \text { min }}{ }^{*}$ rhos

0.08

$\Omega_{T}=-\left(R_{0} / a\right) \mathrm{d} \ln T / \mathrm{d} \rho_{t} 3.5$

Electron beta

$1.5 \%$

q_\{flux surface $\}$

1.8

species

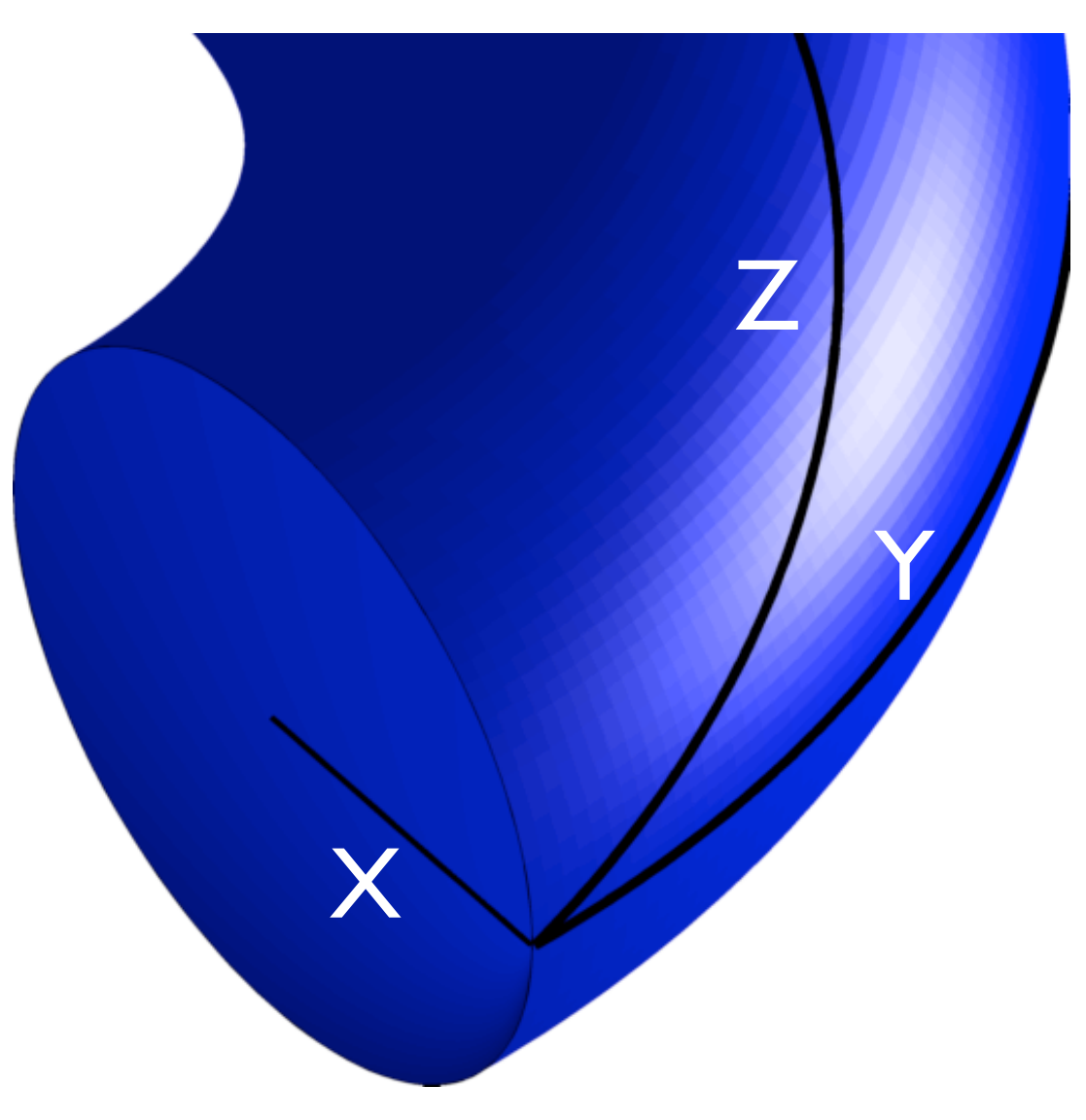




\section{NBI Geometry}

- 4 Injectors

- Tangential geometry

- 5 coordinates

- Real space

- Velocity space

$$
\left(v_{\|}, E\right)
$$

- Weight = beam ionization 